Canadian Journal of Fisheries and Aquatic Sciences

Canadian Science Publishing Journal canadien des sciences halieutiques et aquatiques

\title{
Habitat suitability modeling based on a spatio-temporal model: an example for Cusk in the Gulf of Maine
}

\begin{tabular}{|c|c|}
\hline Journal: & Canadian Journal of Fisheries and Aquatic Sciences \\
\hline Manuscript ID & cjfas-2017-0316.R1 \\
\hline Manuscript Type: & Article \\
\hline Date Submitted by the Author: & 17-Nov-2017 \\
\hline Complete List of Authors: & $\begin{array}{l}\text { Runnebaum, Jocelyn; University of Maine, School of Marine Science } \\
\text { Guan, Lisha; University of Maine, School of Marine Sciences } \\
\text { Cao, Jie; University of Maine, school of marine sciences } \\
\text { O'Brien, Loretta; National Marine Fisheries Service - NOAA, Northeast } \\
\text { Fisheries Science Center } \\
\text { Chen, Yong; University of Maine, }\end{array}$ \\
\hline $\begin{array}{r}\text { Is the invited manuscript for } \\
\text { consideration in a Special } \\
\text { Issue? : }\end{array}$ & N/A \\
\hline Keyword: & $\begin{array}{l}\text { model-based Habitat Suitability Indices, spatio-temporal delta-generalized } \\
\text { linear mixed models, data limited approaches, cusk }\end{array}$ \\
\hline
\end{tabular}


1 Habitat suitability modeling based on a spatio-temporal model: an example for Cusk in the 2 Gulf of Maine

4 Jocelyn Runnebaum ${ }^{1 *}$, Lisha Guan ${ }^{1,2}$, Jie Cao $^{1}$, Loretta O’Brien ${ }^{3}$, and Yong Chen ${ }^{1}$

${ }^{1}$ University of Maine, School of Marine Sciences, 5741 Libby Hall, Orono, ME 04469

7

$8{ }^{2}$ Key Laboratory for Sustainable Utilization of Marine Fisheries, Ministry of Agriculture, Yellow

9 Sea Fisheries Research Institute, Chinese Academy of Fishery Sciences,

10 Qingdao 266071, China

11

$12{ }^{3}$ NOAA/NMFS, Northeast Fisheries Science Center, Woods Hole Laboratory, 166 Water Street,

13 Woods Hole, MA, 02543

14

15

*Corresponding Author: tel: +1 817-793-8541; fax: +1 207-581-4990, email:

16 jocelyn.runnbeuam@maine.edu

17

18 Keywords: Habitat Suitability Index models, spatio-temporal model-based abundance indices, 19 data limited, cusk

20

21

22

23 


\section{Abstract}

25 Habitat use and distribution is a critical aspect in the management and conservation of a species,

26 particularly for those in decline. Habitat suitability indices (HSI) are a common method of

27 habitat mapping dependent on empirical data that can easily lead to misunderstanding the spatio-

28 temporal dynamics of marine species experiencing population decline and density-dependent

29 catchability within surveys. This is especially true when only a single monitoring program with

30 limited spatio-temporal coverage is used. A delta-GLMM was used to combine trawl and

31 longline surveys to predict density estimates for Cusk (Brosme brosme) in un-sampled locations

32 for use in HSIs. Catchability was estimated for longline and trawl gear without having an

33 estimate of area fished for the longline survey. HSIs performed better using model-based density

34 estimates from multiple surveys compared to sample-based abundance indices from a single

35 survey. The increased spatial resolution can better inform the HSIs by providing information

36 where the survey programs did not sample. This study provides a novel approach for integrating

37 data from different monitoring programs for habitat modeling. 


\section{Introduction}

47 Habitat use and distribution is a critical aspect in the management and conservation of a species, particularly for those in decline. Habitat suitability indices (HSI) are a method of assessing

49 relative habitat quality for a species based on abundance at associated environmental conditions for a given location (Brooks 1997; Chen et al. 2009). The HSI can be projected spatially and

51 temporally, providing valuable representation of changes in habitat quality over space and time

52 and potential locations of a species' critical habitat (Chen et al. 2009). Such information is

53 critical for implementing spatially explicit conservation management (Brooks 1997), evaluating

54 shifts in habitat quality over time (Guan et al. 2017; Tanaka and Chen 2016), or understanding

55 the impact of climate variability on suitable habitat distribution (Yu et al. 2016). Recent efforts

56 have been made to improve the predictive capabilities of spatially projected HSIs (Tanaka and

57 Chen 2015; Tanaka and Chen 2016). However, these efforts do not address two critical

58 shortcomings of conventional HSIs: 1) the abundance indices from survey catch data typically

59 incorporated in these models do not account for changes in catchability over a time series; and 2)

60 the commonly used abundance indices, and therefore HSIs, are unable to incorporate surveys

61 from multiple gear types which sample different segments of the population and likely cover

62 different types of habitat. These issues need to be addressed in order to produce an unbiased

63 evaluation of spatio-temporal changes in habitat quality for a species over its distributional 64 range.

65

66 Conventional HSIs have been extensively applied to aquatic species by utilizing abundance

67 indices derived from survey catch data (e.g., catch per unit effort, CPUE; Terrell 1984; Terrell

68 and Carpenter 1997; Morris and Ball 2006). HSIs assume that high density of a species indicates 
69 high quality habitat and that the absence or low density of a species indicates habitat of low

70 value to the species. The use of catch data as a proxy for density assumes that sampled catches

71 truly reflect the density or absence of a species at a given location and are not confounded by

72 stock status, sampling inefficiency and bias. This assumption may be reasonable for species that

73 have relatively constant and high survey catchability over space and time. However, for less-

74 abundant species that are poorly sampled (e.g., low survey catchability or reside in habitat that is

75 not well covered by the survey program), or for which survey catchability has changed over

76 time, conventional HSIs may perform poorly or even produce biased results.

77

78 Conventional HSIs use available data from sampled locations, hereinafter referred to as sample-

79 based HSIs, which are often restricted to the locations of occurrence and typically processed to

80 assume that the samples are representative (i.e., the species is effectively sampled) and are

81 comparable through time (i.e., no changes in sampling distribution and efficiency). Therefore,

82 the sample-based HSIs might not be appropriate in at least the following two situations: 1) the

83 survey misses a significant portion or type of the species' habitat; and 2) sampling efficiency

84 (i.e., catchability) changes over space and/or through time due to density-dependent processes.

85 Density-dependent habitat selection is a likely process for species in decline (MacCall 1990).

86 When a species population is high, individuals move into previously marginal habitat because

87 high quality habitat is saturated; thus, the overall suitability of all occupied habitat declines on

88 average (MacCall 1990). Conversely, as populations decline, individuals retreat to the highest

89 suitable habitat as it becomes less densely occupied and available (MacCall 1990; Hare et al.

90 2012).

91 
92 Another limitation of sample-based HSIs arise when data from multiple surveys are available for

93 a species. Attempts to combine data from multiple surveys face serious difficulties, including

94 quantifying the relative differences of catchability among different sampling gear (i.e., trawl and

95 longline). Such complications often result in discarding data by trimming the survey data to

common surveyed areas and time periods with consistent survey methods, utilizing probability of

97 presence (Grüss et al. 2017; Brotons et al. 2004) or utilizing only one data set when multiples are available (Tanaka and Chen 2016). This is often unsatisfactory due to losses in spatial coverage given that different surveys of different gear types usually sample different areas or habitats. For example, trawl surveys likely do not sample rocky habitat as well as longline surveys. If rocky bottom is one of a species' preferred habitat types, using only trawl surveys for developing HSIs 102 could bias the results.

Cusk (Brosme brosme) in the Gulf of Maine is one species where assessment is difficult using conventional HSIs. It is a data-limited species, with low abundance and low catchability.

106 Catchability of cusk is believed to have declined in the Northeast Fisheries Science Center 107 (NEFSC) spring and fall bottom trawl surveys (BTS) presumably due to declines in stock abundance (Davies and Jonsen 2011; Hare et al. 2012), resulting in density-dependent catchability within the BTS. Additionally, changes to the survey protocols over the time series could influence the catchability of cusk in the BTS. In 2009, the BTS changed the sampling

111 vessel, net type, and tow duration (Politis et al. 2014). The protocol changes in 2009 required the 112 estimation of conversion coefficients for all species to allow for the data to be combined into a 113 continuous time series (Miller et al. 2010). However, low catch numbers and low frequency of 114 occurrence of cusk during the calibration study prevented conversion coefficients to be estimated 
115 for cusk (Miller et al. 2010). An operational stock assessment review in 2009 rejected the 116 proposed analytical assessment and determined that a more representative index of abundance is 117 needed for cusk (NEFSC 2013).

119 Declines in catch of cusk, and similar groundfish species, within the BTS prompted the 120 development of the NEFSC cooperative research bottom longline survey (LLS) in the Western 121 Gulf of Maine to enhance monitoring efforts for data poor and depleted stocks residing in rocky 122 habitat in order to develop a more representative index of abundance (Hoey et al. 2013). The 123 Cusk population is now monitored by these two multispecies survey programs. These two survey 124 programs differ in sampling efficiency, spatial coverage, and duration. Rocky, complex habitat, 125 thought to be utilized by cusk (Collette and Klein-MacPhee 2002; Hare et al. 2012), is not well 126 sampled by the BTS. However, The LLS is designed to sample rocky, complex bottom types 127 more effectively than the BTS covering the same region, due to the nature of the gear. Both 128 survey programs are stratified by depth and overlap in the Western Gulf of Maine.

130 In the case of cusk, the BTS would likely not provide a realistic evaluation of habitat quality 131 because of catch declines over the time series and poor sampling in rocky habitat. Conventional 132 habitat modeling based on either BTS or LLS would likely produce biased results. This study 133 proposes a modeling framework for use with data-limited species such as cusk by combining the 134 bottom trawl survey and the bottom longline survey data to derive model-based density estimates 135 to improve spatial resolution of data for use in HSIs. The results from the model-based HSI are 136 contrasted with those derived from sample-based HSI to test the hypothesis that HSI 
137 performance would improve with the use of higher resolution spatial information from

138 combining multiple surveys and imputing values for un-sampled locations.

\section{Methods}

\subsection{Survey and Environmental Data}

142 Data on cusk abundance are available from the NEFSC spring and fall bottom trawl surveys 143 (1980-2015) and the NEFSC spring and fall bottom longline surveys (2014-2015). The BTS is a 144 demersal, multispecies, depth stratified random survey synoptic of the GOM and GB (Politis et 145 al. 2014). The NEFSC developed a depth stratified random longline survey in the western and central GOM to better sample species that primarily reside in complex habitat (Hoey et al. 2013).

147 Six survey strata were selected for the LLS from ten offshore and four inshore strata from the BTS. This survey also samples in the spring and fall to coincide with the BTS and randomly 149 samples hard bottom sites within each stratum (Hoey et al. 2013). The LLS follows the tidal 150 cycle, with gear deployed one hour before slack tide and fished for two hours. The longline gear 151 is one nautical mile long, with 1000 semi-circle hooks baited with squid set within a three152 nautical mile grid (Hoey et al. 2013).

154 Environmental variables known to impact cusk habitat are depth, temperature, and sediment type 155 (Hare et al. 2012). Cusk have been documented between $18 \mathrm{~m}$ and $1000 \mathrm{~m}$ and are thought to 156 tolerate temperatures between $0{ }^{\circ} \mathrm{C}$ and $14{ }^{\circ} \mathrm{C}$, with the majority of cusk occurring between $6{ }^{\circ} \mathrm{C}$ 157 and $10{ }^{\circ} \mathrm{C}$ in the GOM (Cohen et al. 1990; Collette and Klein-MacPhee 2002). Cusk are 158 hypothesized to prefer rock, gravel, or pebble sediment but are known to inhabit mud areas in the 
159 GOM, excluding smooth sand (Cohen et al. 1990; Collette and Klein-MacPhee 2002). These 160 three environmental variables were used to develop HSIs for the GOM and GB regions.

162 Simulated bottom temperature data (1980-2013) were obtained from the Northeast Coastal 163 Ocean Forecast System (NECOFS) integrated atmosphere-ocean model forecast system for the 164 GOM, GB, and New England Shelf regions. The simulated temperature data were generated 165 from an unstructured Finite-Volume Community Ocean Model (FVCOM) grid for these regions 166 (Beardsley et al. 2013; NECOFS 2013) and averaged over the primary two months when the 167 surveys were conducted.

169 For sample-based HSIs, depth data from the BTS were used. For model-based HSIs depth was 170 extracted from the General Bathymetric Chart of the Oceans (GEBCO) 30 arc-second interval 171 grid. Sediment data were extracted from the United States Geological Survey (USGS) East-Coast 172 Sediment Texture Database (Poppe et al. 2014) using Geographic Information System (GIS). In 173 situ temperature data are not available for the model-based density estimates used in the HSI 174 since these come from model projections. To compare design-based and model-based HSIs the 175 same temperature data were used in both analyses to not confound results by developing HSIs 176 from different types of temperature data (i.e., observed versus simulated). Additionally, a 177 seasonal average can represent the environmental conditions a species would encounter during a 178 season relative to an instantaneous temperature. 179 180 The study area was divided into 5,710 cells $\left(0.05^{\circ} \times 0.05^{\circ}\right)$ for predicting grid-based densities, 181 which were then used to develop model-based HSIs by season. Simulated environmental 
182 variables were assigned to the beginning of the trawl survey location for sample-based HSIs and 183 to the center of $0.05^{\circ} \times 0.05^{\circ}$ grid cells for model-based HSIs.

\subsection{Spatial-Temporal Model for Predicting Species Density}

186 A spatio-temporal delta-generalized linear mixed model (delta-GLMM) initially developed by

187 Thorson et al. (2015) was applied (using the VAST package in R; Thorson et al. 2017; Thorson 188 and Barnett 2017) to data collected from both NEFSC BTS and LLS to estimate cusk density for each season (i.e., spring and fall) from 1980 - 2015, to match the timing of simulated temperature data. This is a two-stage model that ultimately infers population density throughout the study area. Survey data are fit in two stages by: (1) estimating the probability of encountering

192 and catching cusk (i.e., presence/absence) then (2) estimating catches (C) when cusk are present 193 (Thorson et al. 2015).

The first model component estimates the probability $(p)$ of catching at least one of the target 196 species:

$$
\operatorname{Pr}[\mathrm{C}>0]=p
$$

198 The second stage of the model approximates positive catches $(c)$ :

$$
\operatorname{Pr}[\mathrm{C}=\mathrm{c} \mid \mathrm{C}>0]=\operatorname{Gamma}\left(\mathrm{c}, \sigma^{-2}, \lambda \sigma^{2}\right)
$$

200 The probability density function $\operatorname{Gamma}(c, x, y)$ is evaluated at $c$ given a gamma distribution, 201 where $\lambda$ is the expected catch if encountered, and $\sigma$ is the coefficient of variation for positive 202 catches (Thorson and Ward 2014; Thorson et al. 2015). 
204 Spatial autocorrelation is incorporated into the model as a random effect to account for the 205 spatial dependence of species density. Two Gaussian Markov random fields are two included in 206 both stages of the model as a random effect to account for spatial $(\omega)$ and spatio-temporal $(\varepsilon)$ 207 autocorrelation (Thorson et al. 2015). The random fields are approximated at 250 pre-specified 208 knots that are generated based on the proportional density of survey data over the defined 209 domain (i.e., the $0.05^{\circ} \times 0.05^{\circ}$ grid; Thorson et al. 2015). The spatial $(\omega)$ and spato-temporal $(\varepsilon)$ 210 random effects were used in both spring and fall density estimates.

212 Encounter probability $p$ and positive catch rates $\lambda$ are approximated using linear predictors 213 (Thorson et al. 2015):

$$
\begin{aligned}
& p_{i}=\operatorname{logit}^{-1}\left(d_{T_{(i)}}^{(p)}+Q_{i}^{(p)}+\omega_{J_{(i)}}^{(p)}+\varepsilon_{J_{(i)}, T_{(i)}}^{(p)}\right) \\
& \lambda_{i}=w_{i} \exp \left(d_{T_{(i)}}^{(\lambda)}+Q_{i}^{(\lambda)}+\omega_{J_{(i)}}^{(\lambda)}+\varepsilon_{J_{(i)}, T_{(i)}}^{(\lambda)}\right)
\end{aligned}
$$

216 where $p_{i}$ and $\lambda_{i}$ are the expected probabilities of an occupied habitat and positive catches given

217 occupied habitat for sample $i$ at a given location; $d_{T_{(i)}}$ is the average reference density

218 (encounters/positive catch rates) in year $T_{(i)} ; Q_{i}$ is catchability for each survey; $w_{i}$ is the area 219 swept for sample $i ; J_{i}$ is the nearest knot to sample $i ; \omega_{J(i)}$ is a random field accounting for 220 spatially correlated variability at knot $J_{i}$ that is persistent among years; $\varepsilon_{J(i), T(i)}$ is the random

221 field accounting for spatio-temporal correlation at knot $J_{i}$ in year $T_{(i)}$ (Thorson et al. 2015).

222 Spatial and spatial-temporal random fields were used in all models for both seasons.

224 A design matrix with indicator variables for each survey is used to estimate $Q_{i}$. This study 225 assumes the need to estimate three catchability parameters due to the BTS protocol changes in 
2262009 and the inclusion of the LLS. A three-column design matrix was built using

227 ThorsonUtilities with as many rows as observations and reduced to a two-column matrix for

228 identifiability. The 2009 protocol changes cause the intercepts of $Q_{i}$ and $d_{T_{(i)}}$ to be collinear due

229 to a lack of variance in $Q_{i}$ in a given year as a result of two non-overlapping time-blocks in the

230 BTS. To resolve this issue, year effect was modeled via a temporal autocorrelation structure:

$$
\begin{aligned}
& \beta_{1}(t+1) \sim \operatorname{Normal}\left(\rho *_{\beta 1}(t), \sigma_{\beta 1}^{2}\right) \\
& \beta_{2}(t+1) \sim \operatorname{Normal}\left(\rho *_{\beta 2}(t), \sigma_{\beta 2}^{2}\right)
\end{aligned}
$$

where $\rho_{\beta 1}$ and $\rho_{\beta 2}$ are defined as a random walk as specified in the model (Thorson 2017).

234 Catchability is then removed from the model and the underlying species density is predicted at 235 each knot. Grid cells are assigned the density of the nearest knot based on closest Euclidean 236 distance calculated using the Voronoi tool in the PBSmapping package in R (Schnute et al. 237 2013). Density fields are then extracted for each grid cell to use within the HSIs.

Within the delta-GLMM, catch rate was estimated as catch number by area swept (Thorson et al. 2015). Area swept for the BTS tows in the GOM has been standardized as $0.024 \mathrm{~km}^{2}$ for the 241 Bigelow, and $0.038 \mathrm{~km}^{2}$ for the Albatross IV and the Delaware II (NEFSC 2013). The area fished $242\left(A_{i, y} \mathrm{~km}^{2}\right)$ for the LLS is calculated as the distance between the beginning location of the 243 longline and the end of the longline set in $\mathrm{km}(L)$ times an estimated bait plume $(b)$ along the 244 length of the longline for each sample site $(i)$ in a given year $(y)$.

$$
A_{i, y}=L_{i, y} b
$$

246 The bait plume $(b)$ is assumed to be a fixed constant $(0.28 \mathrm{~km})$ for all years and all locations.

247 Evaluation of the impact of varying bait plume sizes on density estimates can be found in 248 Appendix I. 


\subsection{Habitat Suitability Indices}

HSIs quantify the overall habitat quality for a species by evaluating species density associated with each selected environmental variable. Suitability Indices (SI) quantify the relationship between an environmental variable and species abundance at a given location (Terrell 1984; Terrell and Carpenter 1997; Morris and Ball 2006). SIs are then combined either through a geometric mean (GMM) or an arithmetic mean (AMM) to derive an overall habitat suitability index to quantify habitat quality from relatively good (1) to relatively bad (0) (Chen et al. 2009; Tanaka and Chen 2016). HSIs assume that locations with the highest abundance have the highest quality habitat for that organism.

Season-specific HSIs were developed for 1980 - 2013 mean conditions using two different types of abundance indices to compare the performance of model-based HSIs relative to sample-based HSIs. CPUE (i.e., catch number per area swept) from the BTS was used as the abundance index for the sample-based HSIs. Model-based density estimates derived from both the BTS and LLS were extracted for each cell and used in the model-based HSIs. All abundance indices were calculated separately for spring (i.e., April - May) and fall (i.e., October - November). The time series for cusk used in this study is from 1980 to 2015 , however simulated monthly mean temperature data were only available up to 2013 at the time of writing. All data (i.e., observed CPUE and model-based density) were trimmed to 1980-2013 and averaged for the entire time series. NECOFS simulated bottom temperatures (Chen et al. 2006) were averaged by season for the time series. Mean environmental data (i.e., bottom temperature, depth, and sediment type) were extracted for the beginning latitude and longitude for each trawl haul and for each grid center using GIS. 
273 Fisher natural breaks were used to bin the continuous environmental variables of depth and

274 bottom temperature (Bivand 2013; Tanaka and Chen 2016). Sensitivity analyses were conducted

275 to determine both the most appropriate number of bins for each model and the minimum bin size

276 (5-8 bins). Categorical sediment data were extracted from the USGS sediment layer and the nine

277 defined sediment types were used as bins (Poppe et al. 2005).

278

279 For the sample-based HSIs, CPUE for cusk was calculated as catch number at station $(i)$, in 280 season $(s)$, and year $(y)$ per area swept for each vessel (v) (Chang et al. 2012; Tanaka and Chen 2812015 and 2016).

$$
\text { CPUEi }_{\text {sy }}=\frac{{\text { Catch } \text { Number }_{\text {isy }}}_{\text {Area } \text { Swept }_{v}}}{\text { An }}
$$

283 where catch number is the total number of cusk caught per tow and area swept is standardized

284 for each of the three vessels used in the BTS (NEFSC, 2013). For model-based HSIs, mean

285 abundances estimated from the spatio-temporal model were used for each $0.05^{\circ} \times 0.05^{\circ}$ grid cell.

286 The suitability index for bin $(b)$ of environmental variable $(k), \mathrm{SI}_{\mathrm{j}, \mathrm{k}}$, was calculated on a 0.0 to 1.0

287 scale (Chang et al. 2012; Tanaka and Chen 2015 and 2016):

$$
\mathrm{SI}_{b, k}=\frac{\overline{C P U E}_{b, k}-\overline{C P U E}_{k, \min }}{\overline{C P U E}_{k, \max }-\overline{C P U E}_{k, \min }}
$$

289 where $\overline{C P U E_{b, k}}$ is the average CPUE over all sampled stations within bin $b$ for each

290 environmental variable $k$ (Tanaka and Chen, 2015 and 2016). These SI values were then

291 averaged by an arithmetic mean (AMM) and a geometric mean (GMM).

$$
\begin{aligned}
H S I_{A M M} & =\frac{\sum_{i=1}^{n} S I_{k}}{n} \\
H S I_{G M M} & =\prod_{i=1}^{n} S I_{k}{ }^{1 / n}
\end{aligned}
$$


294 where all $\mathrm{SI}_{\mathrm{i}}$ represent equally weighted SI values for the $k^{\text {th }}$ environmental variable and $n$ is the 295 number of environmental variables included.

297 The sample- and model-based HSIs were based on empirical data from the BTS survey and 298 modeled density estimates, respectively. Due to the limitation of sample-based HSI, the CPUE 299 used was restricted to only the spring and fall BTS survey. However, model-based HSIs 300 incorporated density estimates derived from both the BTS and LLS. Density estimates are 301 extrapolated over the grid cells based on the abundance estimates for the nearest knot. The $0.05^{\circ}$ $302 \times 0.05^{\circ}$ grid size was used to increase spatial resolution for environmental variables over the 303 entire survey area.

\section{Results}

\subsection{Spatial-Temporal Model for Predicting Species Density}

307 Varying estimates of area fished for the LLS were also tested to evaluate the relative impact of 308 bait plumes on abundance estimates. The tested values of $b$ to estimate area fished (i.e., $0.28 \mathrm{~km}$, $3090.56 \mathrm{~km}, 1.12 \mathrm{~km}$ ) showed to have no impact on density estimates and annual indices of 310 abundance because the catchability coefficient could account for differences in catch rate

311 (Appendix I). This makes it possible to combine two different gear types without needing to 312 know the size of the bait plume for the longline survey.

314 Density plots for annual species distribution indicate that the cusk population is densest in the 315 central GOM, with annual variability (Figure 1). Cusk density has constricted over the time316 period, with lower densities predicted inshore in both seasons later in the time series (Figure 1).

317 From 1980 to 1993 cusk density is highest in the time series, with a slight decrease in density 
318 particularly in the offshore (yellow and red regions, Figure 1). Cusk population density from

3191994 to 2007 remains relatively constant in the spring and the fall. Starting in 2008 to the end of

320 the time series shows low density levels particularly in the inshore regions. Over the time series

321 density around Georges Bank (i.e., the southernmost extent of the plots) shows a steady decline

322 in cusk abundance, predominately in the spring and somewhat in the fall, over the entire time

323 series (Figure 1). Pearson residual plots suggest there is no significant spatio-temporal pattern in 324 the residuals (Figure 1).

\subsection{Habitat Suitability Index Models}

\subsubsection{Sample-Based HSI}

328 Sample-based SIs were derived from observed CPUE from the BTS using simulated temperature and sediment data and observed average depths. Simulated seasonal mean bottom temperatures were compared to observed instantaneous bottom temperatures from the BTS when available

(Figure 2). The instantaneous observed temperatures were more variable (spring: $1.35^{\circ} \mathrm{C}$ to 12.30

${ }^{\circ} \mathrm{C}$; fall: $4.47{ }^{\circ} \mathrm{C}$ to $19.20^{\circ} \mathrm{C}$ ) compared to simulated temperatures (spring: $4.29^{\circ} \mathrm{C}$ to $7.64{ }^{\circ} \mathrm{C}$; fall:

$3336.66{ }^{\circ} \mathrm{C}$ to $14.09^{\circ} \mathrm{C}$; Figure 2). Observed mean depths were used to build sediment sample-based

334 SIs. Observed depths were compared to simulated depths at the location of sampling and were 335 more variable at deeper depths than at shallower depths but were well correlated (Figure 3).

337 Assuming the simulated temperature represents mean conditions that cusk would experience 338 during each season, the preferred mean temperatures for cusk were between $7.05^{\circ}$ to $7.63^{\circ} \mathrm{C}$ in 339 the spring and 8.14 to $8.72^{\circ} \mathrm{C}$ in the fall (Figure 4). Cusk preferred depths between $189 \mathrm{~m}$ to $340224 \mathrm{~m}$ in the spring and $192 \mathrm{~m}$ to $227 \mathrm{~m}$ in the fall (Figure 4). Bedrock was the most preferred 
341 sediment type followed by a combination of sand, silt, and clay in the spring sample-based HSI

342 (Figure 4) and combinations of clay, silt, and sand in the fall sample-based HSI (Figure 4).

344 HSIs assume that habitat quality increases with density. A simple linear regression between 345 abundance and HSI was used to test this assumption. Sample-based HSIs for the spring and fall 346 did not show a clear relationship between density and habitat quality (Table 1). Linear 347 regressions between CPUE and sample-based HSIs in the spring showed a significant 348 relationship (p-value $<0.01$ ) but the models failed to fit the data well (e.g., spring AMM, CPUE $349 \mathrm{R}^{2}<0.072$; fall AMM, CPUE $\mathrm{R}^{2}<0.05$; Table 1).

\subsubsection{Model-Based HSI}

352 Model-based SIs were derived from density field estimates from the delta-GLMM (spring and 353 fall, $b=0.28 \mathrm{~km}$ ). Model-based bottom temperature SI curves found $6.87^{\circ}$ to $7.25^{\circ} \mathrm{C}$ as the most 354 suitable temperatures in the spring and $8.07^{\circ}$ to $8.68^{\circ} \mathrm{C}$ in the fall (Figure 4). SI depth curves for 355 abundance indices derived from both data sets, showed that $161 \mathrm{~m}$ to $208 \mathrm{~m}$ was the most

356 preferred depth range in both the spring and fall. For all spring and fall model-based HSI models, 357 the most preferred sediment type was a combination of sand, silt, and clay in both the spring and 358 fall (Figure 4).

\subsubsection{Comparison Between Sample- and Model-Based HSI}

361 The model-based SIs and sample-based SIs have similar results in habitat use for both the spring

362 and the fall, with the exception of sediment use. Both the model-based and sampled-based

363 bottom temperature SI curves indicate cusk were caught in slightly warmer waters in the fall but 
364 prefer temperatures around $7^{\circ} \mathrm{C}$ in the spring and $8^{\circ} \mathrm{C}$ in the fall (Figure 4). The model-based 365 depth SI curves showed cusk were associated with depth ranges between $2 \mathrm{~m}$ and $877 \mathrm{~m}$ in the 366 spring and fall compared to BTS observed depth ranges in the spring (22 to $368 \mathrm{~m}$ ) and fall (20 367 to $412 \mathrm{~m}$ ). Both model-based depth SI curves showed $161 \mathrm{~m}$ to $208 \mathrm{~m}$ as the most preferred depth 368 ranges in the spring and fall (Figure 4). These preferred depth ranges are shallower than the 369 preferred depth ranges estimated (189 $\mathrm{m}$ to $224 \mathrm{~m}$ in the spring and $192 \mathrm{~m}$ to $227 \mathrm{~m}$ in the fall) 370 by the sample-based depth SI, but show the same pattern of preferring deeper depths in the 371 spring and shallower depths in the fall (Figure 4). Pearson correlations (r) indicate temperature 372 and depth were positively correlated in the spring for both model-based $(r=0.61)$ and sample373 based $(\mathrm{r}=0.28)$ HSIs and negatively correlated in the fall for model based $(\mathrm{r}=-0.71)$ and 374 sample-based HSIs $(r=-0.31)$.

375

376 The model-based GMM HSIs had higher coefficient of determination $\left(\mathrm{R}^{2}\right)$ and correlation 377 coefficients for both seasons in all models except in the fall sample-based HSI (Table 1). Model378 based HSIs derived only from the BTS were statistically significant $(\mathrm{p}<0.001)$ with an $\mathrm{R}^{2}$ of $3790.3836(\mathrm{AMM})$ and $0.4366(\mathrm{GMM})$ for the spring and 0.2927 (AMM) and $0.3041(\mathrm{GMM})$ for 380 the fall (Table 1, modeled BTS only). Model-based HSIs predicted habitat quality well relative to 381 survey catch rates (Figure 8).

382

383 The preferred range of each environmental variable for sample-based HSIs is limited by the 384 sampling locations. The model-based sediment SI histograms derived for both the spring and fall 385 indicated a mixture of sand, silt, and clay to be the most preferred sediment (Figure 4). Most of 386 the cusk catches in both the spring and fall BTS were in these sediment types (Figure 5). For 
387 sample-based sediment, SI histograms indicated that bedrock was the most important sediment 388 type in the spring and gravel the third most important for the fall (Figure 4). However, for the 389 entire time series (1980 - 2013) one cusk was caught in bedrock and two in gravel in the spring, 390 and in the fall zero were caught in bedrock and six were caught in gravel (Figure 5). Similarly, sample-based HSIs depicted a narrower depth range and preferred depth relative to model-based

392 HSIs (Figure 4). The NEFSC trawl survey sampling locations were shallower (e.g., $22 \mathrm{~m}$ $393388 \mathrm{~m}$ in the spring and $20 \mathrm{~m}$ to $516 \mathrm{~m}$ in the fall; Figure 6) relative to simulated depths available 394 to the model-based HSIs (i.e., $2 \mathrm{~m}$ to $877 \mathrm{~m}$ ).

The SI for each variable was removed to determine the relative importance of that variable to the

397 overall HSI, the bigger the decrease in $\mathrm{R}^{2}$ the more important that variable is to habitat

398 suitability. For sample-based HSIs, temperature was the biggest driver in habitat suitability in the 399 spring while sediment was the biggest driver in the fall (Table 1). However, for the fall sample400 based GMM HSI the $\mathrm{R}^{2}$ increased when depth was removed indicating this variable is not 401 important within that model potentially due to the negative correlation with temperature. For 402 model-based HSIs, temperature was the biggest driver in the spring, and depth was the biggest 403 driver in the fall (Table 1).

4054 Discussion

406 Comparisons between model-based and sampled-based HSIs indicate that fully utilizing all 407 available survey data changed the perception of depth and sediment preferences of cusk because 408 of the imputed estimates for un-sampled locations. Thus, model-based density estimates that 409 incorporated data from multiple gear types can overcome the sampling bias of trawl surveys for 
410 species that associate with complex habitats. HSIs were better able to predict an increase in

411 habitat quality with increasing density with the use of model-based abundance estimates

412 (Table 1).

\subsection{Spatial-Temporal Model for Predicting Species Density}

415 Bottom trawl surveys are likely to produce biased estimates of abundance if the species' spatial 416 distribution has changed over time and if gear performance varies by the habitat that the target 417 species associates with over time (Thorson et al. 2013; Kotwicki et al. 2014). The decline in the 418 cusk population likely reduced catchability in the BTS due to the population constricting to 419 rocky habitat not accessible to the BTS (Davies and Jonsen 2011; Hare et al. 2012), resulting in a 420 decreased probability of successful sampling. Annual density field estimates and subsequent 421 abundance indices (Appendix I) based only on the BTS are therefore likely biased due to 422 density-dependent and time-varying catchability.

424 The LLS was incorporated into the model-based abundance estimates to compensate for density425 dependent processes within the BTS. Density-dependent processes cause systematic biases in 426 BTS indices of abundance which can lead to large errors in estimating species' spatial

427 distribution (Thorson et al. 2013; Kotwicki et al. 2014). Density fields derived from both surveys 428 have similar patterns in spatial distribution (i.e., high density versus low density) as those 429 derived from the BTS only (Appendix I). However, there are finer resolution changes throughout 430 the time series when the LLS is incorporated, particularly in fall. This qualitatively supports the 431 hypothesis that cusk catches in the BTS have experienced density-dependent catchability. The 432 LLS is thought to be a better method of sampling groundfish species that are not well sampled by 
433 mobile bottom tending gear (Hoey et al. 2013). Biases in predicting a species' spatial distribution

434 can be addressed through the incorporation of data from surveys that sample habitats differently.

436 Accounting for differences in catchability has been shown to produce abundance estimates with 437 reduced variability when multiple vessels are involved (Thorson and Ward 2014). The delta438 GLMM (Thorson et al. 2015) estimated catchability for the LLS as well as before and after the 4392009 protocol changes by considering these two time-blocks to be surveys with separate 440 catchability parameters. To test the sensitivity of the model to catchability estimates for the LLS 441 three different values $b$ to estimate area fished (i.e., $0.28 \mathrm{~km}, 0.56 \mathrm{~km}, 1.12 \mathrm{~km}$; Appendix I).

442 Varying values of area fished had no impact on the density estimates and estimated catchability

443 in the model accounted for differences in area fished (Appendix I). Finer temporal changes in 444 catchability (e.g., year-specific changes) were not considered but may be useful to incorporate in 445 the future or for species with similar circumstances. However, a random year effect was included 446 to account for time-varying catchability for the BTS before and after 2009 and for the LLS.

448 Including the longline survey when deriving density estimates changes the abundance estimates 449 in the most recent years (Appendix I). As the LLS time-series increases, the perceived systematic 450 bias in abundance estimates from the BTS can be tested and addressed. The LLS would need to 451 be conducted at the same locations as the BTS tows to compare catch rates from the two surveys 452 to fully understand the consequences of spatially varying catchability (Thorson et al. 2013). As 453 species decline and are more likely distributed in areas with complex bottom, they become less 454 accessible to trawl gear. Longline surveys can sample these areas to provide additional data 455 complementary to the data derived in mobile trawl surveys. This study shows how data from 
456 longline and trawl gear can be combined within a modeling framework and how the HSI benefits 457 from doing so.

\subsection{Habitat Suitability Index Models}

460 Overall, the model-based HSIs could better capture variance in abundance at different levels of 461 habitat quality compared to sample-based HSIs. The increased spatial resolution of density 462 estimates (Figure 1) can provide information for a species' use of habitat without perfect 463 sampling coverage or low catch rates. Utilizing model-based abundance indices changes the 464 perception of habitat use through two mechanisms. First, inclusion of the LLS provides data for 465 the model outside of where the BTS sampled. The use of two gear types within the model-based 466 density estimates provides increased data on habitat use because of the ability to sample in 467 different sediment types. By utilizing all available data, the subsequent HSIs were better 468 informed than both the sample-based HSIs and model-based HSIs that only used the BTS to 469 predict abundance. Second, the estimated density fields by grid provides information where the 470 LLS and/or BTS did not sample. Increasing the spatial resolution of density estimates and the 471 interaction with environmental variables is believed to be the primary driver in improving the 472 HSIs.

473

474 Conventional sample-based HSIs are likely influenced by the availability of sampled data, as 475 evidenced by the poor performance of sample-based HSIs relative to model-based HSIs 476 (Table 1). The sampled depth ranges in spring (22 $\mathrm{m}-368 \mathrm{~m})$ and fall (20 $\mathrm{m}$ and $412 \mathrm{~m})$ BTS 477 limit what data are informing the HSI. Additionally, sampling frequency at various depths 478 (Figure 6) and sediment types (Figure 5) influences the performance of HSIs. The limited data 
479 available for sample-based HSIs prompted the need for improving the spatial resolution of

480 species' density estimates and environmental variables over a study area. The improved spatial

481 resolution of environmental variables (i.e., per grid cell) overcomes the limitations of empirical

482 data availability at a sampled location by providing finer-scale resolution of a species'

483 interaction with these environmental variables. The improvements in spatial resolution also

484 provides the ability to compare the overlap in habitat distribution of two species using the same spatial grid, a critical aspect of bycatch mitigation and species conservation.

487 However, the frequency of observations at each range within the environmental variables is 488 influential on the HSI outcome. This was most notable for evaluating sediment type SIs.

489 Sediment SIs for sample-based HSIs indicated bedrock to be the most important sediment type in 490 the spring and a mixture of sand, clay, and silt in the fall (Figure 4). A mixture of sand, silt, and 491 clay was the most important sediment type for the spring and fall model-based HSIs and bedrock 492 was among the lowest (Figure 4). The sample-based SI sediment curves are likely biased due to 493 low catch rates in more complex sediment types (Figure 5). Few cusk were caught in bedrock in 494 the BTS (1 in spring, 0 in fall) and in gravel (2 in spring and 6 in fall) between 1980-2013

495 (Figure 5). The model-based HSIs provide abundance estimates for areas not directly observed in 496 the survey, allowing for a different understanding of how cusk might be utilizing different 497 sediment types. Model-based abundance estimates associated cusk with bedrock (20 times in the 498 spring, 21 in the fall) and gravel (95 times in the spring, and 88 times in the fall). Cusk are 499 thought to predominately reside on hard bottom (i.e., bedrock and gravel; Collette and Klein500 MacPhee 2002), making the model-based abundance estimates associated with hard bottom more 501 realistic with regard to presumed cusk behavior. The LLS could provide catch data for sediment 
502 types that the BTS cannot provide consistent data for, which provides more data for spatially

503 explicit density estimates over the time series (Figure 1). Model-based abundance estimates that

504 incorporate the LLS are thought to improve data quality for use in habitat mapping by better

505 informing the model in areas not well sampled by the BTS. However, the frequency of

506 observations for both the sample-based and model-based HSIs are likely influencing the results.

507

508 This study includes the three most important variables for this species reported in the literature

509 (Collette and Klein-MacPhee 2002; Hare et al. 2012), reasonably capturing variability in habitat

510 distribution with only three variables in the model-based HSIs. Temperature, depth, and

511 sediment were equally weighted within the sample-based and model-based HSIs, preliminary

512 analyses indicated that SI weighting did not improve the HSIs. However, the relative

513 contribution of each variable is likely not equal (Table 1). For model-based HSIs, temperature

514 was the most influential variable in defining habitat suitability the spring while depth was the

515 most influential in fall (Table 1). Temperature is hypothesized to be a significant contributor to

516 habitat suitability for cusk (Hare et al. 2012), but this evaluation indicates that variable

517 importance may shift between seasons. However, mean seasonal temperatures and depth are

518 positively correlated in the spring and negatively correlated in the fall, making it difficult to

519 separate the relative influence of each variable. Even though depth and temperature are

520 correlated, both variables contribute to defining habitat suitability (Table 1). Cusk spawn in the

521 spring (Collette and Klein-MacPhee 2002) and may be seeking preferred temperatures in

522 shallower waters during spawning events while moving to deeper waters in the fall seeking

523 colder temperatures. 


\subsection{Model Limitations}

HSI models are a relative index traditionally built from empirical data. These models cannot account for uncertainty in their estimates. Using model-output as model-input can incorporate unaccounted uncertainty that can be magnified within the second model (Brooks and Deroba 2015). However, the HSI model used in this study is not able to account for uncertainty no matter if modeled or empirical data are used. Pearson residual plots indicate the incorporation of the LLS increased uncertainty due to the large differences in catch numbers between the two survey programs. HSIs assume that habitat suitability increases linearly with abundance. With limited data for sample-based HSIs, linear regressions were the most reliable method of testing model performance. Future research should focus on: 1) building model-based HSIs with part of the data and using the remaining data to develop HSIs (Tanka and Chen 2016); 1) using the delta-GLMM to evaluate habitat preference; and/or 2) using model-based abundance estimates that have an associated uncertainty in abundance to account for uncertainty within habitat modeling.

This study did not assume that Cusks' preferences for sediment, depth, and temperature change on an annual basis. The relationship between mean abundance and each of these variables was assumed to be constant during 1980 to 2013. HSI models are typically used for understanding a species' response to changes in habitat (Terrell and Carpenter 1997). Many HSI models assume that an organism's habitat preference does not change on an annual basis; distribution might, but the underlying relationship does not (Chen et al. 2011; Tanaka and Chen 2015; Guan et al. 2017). For the HSI models in this study to provide such insight, annual predictions would need to be made. 
549 This study does not evaluate age or size dependent processes in habitat selection. Cusk caught in the LLS $(2014$ - 2015) have a similar median size $(57 \mathrm{~cm})$ but narrower length range $(30-84 \mathrm{~cm})$

551 compared to cusk caught in the spring and fall BTS, which had a much wider length range (11-

$55294 \mathrm{~cm})$ but similar median size $(58 \mathrm{~cm})$. Cusk caught from 2014 to 2015 in the BTS have a

553 smaller median size $(50 \mathrm{~cm})$ than cusk caught over the included time series $(1980-2015)$ and in

554 the longline survey (Figure 7). These size differences are expected given the two gear types have 555 different selectivities, however there are only sixty-nine cusk caught in the BTS in 2014 and

556 2015. The average size at maturity for cusk in the Scotian Shelf area is $50 \mathrm{~cm}$ for males and 557 females combined (COSEWIC 2003). These two gear types catch predominantly mature

558 individuals; a quarter of the catch is below $40 \mathrm{~cm}$. Based on these size distributions, it is assumed 559 that the habitat suitability indices represent adult habitat.

561 Annual density estimates can be used to build annual HSI models to evaluate how habitat quality

562 has changed over a time series. The impacts of a changing climate can be better understood and 563 predicted for the future by understanding the ideal range of each environmental variable.

564 Currently, the delta-GLMM cannot predict potential or realized habitat (Grüss et al. 2017) as it 565 can be from the HSI. However, several environmental factors influence a species' habitat use 566 that might not be fully captured by the environmental variables used within an HSI. Even if all 567 known environmental drivers were incorporated there would likely be unaccounted for 568 stochasticity within habitat distribution and species abundance. The spatio-temporal delta569 GLMM captures spatial and spatial-temporal variability of species density through the random 570 effects in the model (Thorson et al. 2015). The stochasticity of species distribution, that is 
571 unlikely to be captured by any given variable or set of variables in the HSI is presumed to be

572 captured by first predicting species density within the delta-GLMM. Environmental covariates

573 incorporated into the delta-GLMM to predict species density could be used to evaluate the

574 suitable range of environmental variables to understand species' habitat preferences as this

575 model progresses.

576

5775 Conclusion

578 The delta-GLMM provided a means of generating modeled abundance that reflects spatial

579 heterogeneity in species density and utilizes all available survey data. The incorporation of

580 different gear types to estimate abundance can, in part, overcome systematic density-dependent

581 sampling biases that are seen in trawl surveys when a species' abundance contracts to habitat that

582 is not effectively sampled by the survey. Spatially explicit abundance estimates provide a means

583 of evaluating the habitat suitability, by providing estimates in areas that were not directly

584 sampled by the survey. The increased spatial resolution of abundance data improved the habitat

585 suitability models in this study. A delta-GLMM offers a method of providing abundance

586 information for areas not sampled by survey programs and for species caught in low numbers.

587

588

589

590

591

592

593 


\section{Acknowledgments}

595 We would like to thank Dr. James Thorson of the Northwest Fisheries Science Center for his 596 reviews and help with the delta-GLMM. Thank you to the NEFSC internal reviewer for

597 thoughtful comments for improving the second version of this manuscript. We would like to 598 thank the anonymous reviewer for the first version of this manuscript. We are grateful for Dr.

599 Dave McLeroy at the NOAA Northeast Fisheries Science Center for collecting the longline 600 survey data. We also thank Nancy McHugh from the Ecosystems Surveys Branch, NOAA

601 Fisheries for providing the NEFSC bottom trawl survey data. We appreciate Kisei Tanaka of the 602 University of Maine for his advice on habitat mapping. The NECOFS simulated temperature data 603 in this study were from NERACOOS, Massachusetts Fishery Institution, and MIT Sea Grant 604 College programs. This research was funded by NOAA Saltonstall-Kennedy grant number 605 NOAA NA14NMF4270029.

606 607 608 609 610 611 612 613 614 615 616 
617

618

619

620

621

622

623

624

625

626

627

628

629

630

631

632

633

634

635

636

637

638

639

\section{References}

Beardsley, R.C., Chen, C., and Xu, Q. 2013. Coastal flooding in Scituate (MA): A FVCOM study of the 27 December 2010 nor'easter. Journal of Geophysical Research - Oceans, 118: 6030-6045.

Bivand, R. 2013. R Package “classInt": choose univariate class interval (Ver 0.1-21). R-Project. Accessed October 23, 2014. Available at: http://cran.rproject.org/web/packages/classInt/index.html.

Brooks, R. P. 1997. Improving Habitat Suitability Index Models. Wildlife Society Bulletin, 25(1): $163-167$.

Brooks, E.N., Deroba J.J. 2015. When "data" are not data: the pitfalls of post hoc analyses that use stock assessment model output. Canadian Journal of Fisheries and Aquatic Sciences. 72(4): 634-641.

Brotons, L., Thuiller, W., Arauijo, M. B. and Hirzel, A. H. 2004. Presence-absence versus presence-only modelling methods for predicting bird habitat suitability. Ecography 27: 437-448.

Chang, Y., Sun, C., Chen, Y., and Yeh, S. 2012. Habitat suitability analysis and identification of potential fishing grounds for swordfish, Xiphias gladius, in the South Atlantic Ocean. International Journal of Remote Sensing. 33(23): 7523-7541.

Chen, C., Beardsley, R. C., and Cowles, G. 2006. An unstructured grid, finite-volume coastal ocean model (FVCOM) system. Special Issue entitled "Advance in Computational Oceanography". Oceanography. 19: 78-89

Chen, X.J., Li, G., Fend, B. and Tian, S.Q. 2009. Habitat suitability of chub mackerel (Scomber japonicus) in the East China Sea. Journal of Oceanography. 65: 93-102. 
640 Chen, X., Tian, S., Liu, B., and Chen, Y. 2011. Modeling a habitat suitability index for the 641 eastern fall cohort of Ommastrephes bartramii in the central North Pacific Ocean. Chinese Journal of Oceanology and Limnology. 29(3): 493-504.

Cohen, D.M., Inada, T., Iwamoto, T. and Scialabba, N. 1990. Gadiform fishes of the world. 644 Food and Agriculture Organization of the United Nations Species Catalogue. Fir/S125.

645 Vol. 10.

646

Collette, B.B. and Klein-MacPhee, G. 2002. Bigelow and Schroeder's Fishes of the Gulf of Maine. Washington, DC: Smithsonian Institution

648 649 650 651 652 653 654 655 656 657 658 659 660 661 662

Committee on the Status of Endangered Wildlife in Canada (COSEWIC). 2003. Assessment and Status Report on the Cusk (Brosme brosme) in Canada. May 2003.

Davies, T. D., and Jonsen, I. D. 2011. Identifying nonproportionality of fishery-independent survey data to estimate population trends and assess recovery potential for cusk (Brosme brosme). Canadian Journal of Fisheries and Aquatic Sciences. 68(3): 413-425.

General Bathymetric Chart of the Oceans (GEBCO). 2014. The GEBCO_2014 Grid, version 20150318, http://www.gebco.net.

Grüss A, Sagarese SR, Karnauskas M, Walter III JF, Babcock EA, Drexler M. 2017. Ontogenetic spatial distributions of red grouper (Epinephelus morio) and gag grouper (Mycteroperca microlepis) in the Northern Gulf of Mexico. Fisheries Research 193: 129-142.

Guan, L., Chen, Y., and Wilson, J. A. 2017. Evaluating spatio-temporal variability in the habitat quality of Atlantic cod (Gadus morhua) in the Gulf of Maine. Fisheries Oceanography. 26(1): 83-96.

Hare, J. a., Manderson, J. P., Nye, J. A., Alexander, M. A., Auster, P. J., Borggaard, D. L., and Biegel, S. T. 2012. Cusk (Brosme brosme) and climate change: assessing the threat to a 
candidate marine fish species under the US Endangered Species Act. ICES Journal of Marine Science. 69(10): 1753-1768.

Hoey, J., O’Brien, L., Natanson, L., McMlroy, D., Manderson, J., Borggaard, D., and Silva, R. 2013. Development of a bottom longline survey for stocks associated with complex rocky habitat in the western and central Gulf of Maine, including several data poor stocks that are ESA species of concern. NEFSC \& NERO PROPOSAL 2013 Cooperative Research Solicitation. Available from: National Marine Fisheries Service, 166 Water Street, Woods Hole, MA 02543-1026.

Kotwicki, S., Ianelli, J. N., and Punt, A. E. 2014. Correcting density dependent effects in abundance estimates from bottom trawl surveys. ICES Journal of Marine Science. 71: $1107-1116$.

Lokkeborg, S., B. L. Olla, W.H. Pearson, and M. W. D. 1995. Behavioural responses of sablefish, Anoplopoma fimbria, to bait odour. Journal of Fish Biology, 46, 142-155.

MacCall, A.D. 1990. Dynamic geography of marine fish populations. University of Washington Press. Seattle, WA, USA.

Miller TJ, Das C, Politis PJ, Miller AS, Lucey SM, Legault CM, Brown RW, and Rago, PJ. 2010. Estimation of Albatross IV to Henry B. Bigelow calibration factors. Northeast Fish Sci Cent Ref Doc. 10-05; 233 p. Available from: National Marine Fisheries Service, 166 Water Street, Woods Hole, MA 02543-1026.

Morris, L., and Ball, D. 2006. Habitat suitability modelling of economically important fish species with commercial fisheries data. ICES Journal of Marine Science. 63(9): 15901603. 
685 Northeast Coastal Ocean Forecasting System (NECOFS). 2013. Northeast Coastal Ocean

686

687

688

689

690

691

692

693

694

695

696

697

698

699

700

701

702

703

704

705

706

707

Forecasting System Main Portal.

http://fvcom.smast.umassd.edu/research_projects/NECOFS.

Northeast Fisheries Science Center (NEFSC). 2013. 55th Northeast Regional Stock Assessment Workshop (55th SAW) Assessment Report. US Dept Commer, Northeast Fish Sci Cent Ref Doc. 13-11; 845 p. Available from: National Marine Fisheries Service, 166 Water Street, Woods Hole, MA 02543-1026, or online at http://www.nefsc.noaa.gov/nefsc/publications/

Politis, P. J., Galbraith, J. K., Kostovick, P., and Brown, R. W. 2014. Northeast Fisheries Science Center Bottom Trawl Survey Protocols for the NOAA Ship Henry B. Bigelow.

Poppe, L.J., McMullen, K.Y., Williams, S.J., and Paskevich, V.F., eds. 2014. USGS east-coast sediment analysis: Procedures, database, and GIS data (ver. 3.0, November 2014): U.S. Geological Survey Open-File Report 2005-1001, http://pubs.usgs.gov/of/2005/1001/.

Schnute, J.T., Boers, N., and Haigh, R. 2015. Mapping Fisheries Data and Spatial Analysis Tools. R package. Fisheries and Oceans Canada.

Tanaka, K., and Chen, Y. 2015. Spatiotemporal variability of suitable habitat for American lobster (Homarus Americanus) in Long Island Sound. Journal of Shellfish Research. 34(2): 531-543.

Tanaka, K., and Chen, Y. 2016. Modeling spatiotemporal variability of the bioclimate envelope of (Homarus americanus) in the coastal waters of Maine and New Hampshire. Fisheries Research. 177: 137-152.

Terrell, J.W. 1984. Proceedings of a workshop on fish habitat suitability index models. Biological Report. 85: 393. 
Terrell, J. W., and Carpenter, J. 1997. Selected Habitat Suitability Index Model Evaluations. Information and Technology Report, USGS/BDR/IT -- 1997-0005.

Thorson, J.T., Clarke, M.E., Steward, I.J., and Punt, A.E. 2013. The implications of spatially varying catchability on bottom trawl surveys of fish abundance: a proposed solution involving underwater vehicles. Canadian Journal of Fisheries and Aquatic Sciences. 7: 294-306.

Thorson, J. T., and Ward, E. J. 2014. Accounting for vessel effects when standardizing catch rates from cooperative surveys. Fisheries Research. 155: 168-176.

Thorson, J.T., Shelton, A.O., Ward, E.J., and Skaug, H.J. 2015. Geostatistical delta-generalized linear mixed models improve precision for estimated abundance indices for West Coast groundfishes. ICES Journal of Marine Sciences. 72(5): 1297-1310.

Thorson, J.T. and Barnett, L.A.K. 2017. Comparing estimates of abundance trends and distribution shifts using single- and multispecies models of fishes and biogenic habitat. ICES Journal of Marine Science. 74(5): 1311-1321.

Thorson, J.T. 2017. VAST user manual. https://github.com/JamesThorson/VAST/blob/master/examples/VAST user manual.pdf . Accessed 5/19/2017.

Yu, W., Qian, Y., Chen, X., and Chen, Y. 2016. Modelling the effects of climate variability on habitat suitability of jumbo flying quid, Dosidicus gigas, in the Southeast Pacific off Peru. ICES Journal of Marine Science. 73(2): 239-24.

Zhou, S., Klaer, N. L., Daley, R. M., Zhu, Z., Fuller, M., and Smith, A. D. M. 2014. Modelling multiple fishing gear efficiencies and abundance for aggregated populations using fishery or survey data. ICES Journal of Marine Science. 71(9): 2436-2447. 
731 Table 1. HSI model comparisons. Linear regression results between abundance and arithmetic

732 mean (AMM) and geometric mean (GMM) HSI results and Pearson's correlation coefficient

733 (estimated in R).

\begin{tabular}{|c|c|c|c|}
\hline Model & $\mathbf{R}^{2}$ & $\begin{array}{l}\text { Correlation } \\
\text { Coefficient }\end{array}$ & $\begin{array}{l}\text { p-value of } t- \\
\text { test for slope } \\
\text { coefficient }\end{array}$ \\
\hline Spring AMM, model-based HSI & 0.384 & 0.619 & $<0.01$ \\
\hline w/o bottom temperature & 0.266 & 0.516 & $<0.01$ \\
\hline w/o depth & 0.362 & 0.602 & $<0.01$ \\
\hline w/o sediment & 0.359 & 0.599 & $<0.01$ \\
\hline Spring GMM, model-based HSI & 0.437 & 0.661 & $<0.01$ \\
\hline w/o bottom temperature & 0.280 & 0.529 & $<0.01$ \\
\hline w/o depth & 0.419 & 0.647 & $<0.01$ \\
\hline w/o sediment & 0.406 & 0.637 & $<0.01$ \\
\hline Spring AMM, sample-based HSI & 0.073 & 0.270 & $<0.01$ \\
\hline w/o bottom temperature & 0.054 & 0.232 & $<0.01$ \\
\hline w/o depth & 0.070 & 0.264 & $<0.01$ \\
\hline w/o sediment & 0.060 & 0.245 & $<0.01$ \\
\hline Spring GMM, sample-based HSI & 0.085 & 0.291 & $<0.01$ \\
\hline w/o bottom temperature & 0.058 & 0.241 & $<0.01$ \\
\hline w/o depth & 0.083 & 0.288 & $<0.01$ \\
\hline w/o sediment & 0.067 & 0.259 & $<0.01$ \\
\hline Fall AMM, model-based HSI & 0.293 & 0.541 & $<0.01$ \\
\hline w/o bottom temperature & 0.262 & 0.512 & $<0.01$ \\
\hline w/o depth & 0.219 & 0.468 & $<0.01$ \\
\hline w/o sediment & 0.280 & 0.529 & $<0.01$ \\
\hline Fall GMM, model-based HSI & 0.304 & 0.551 & $<0.01$ \\
\hline w/o bottom temperature & 0.280 & 0.529 & $<0.01$ \\
\hline w/o depth & 0.222 & 0.471 & $<0.01$ \\
\hline w/o sediment & 0.293 & 0.542 & $<0.01$ \\
\hline Fall AMM, sample-based HSI & 0.044 & 0.211 & $<0.01$ \\
\hline w/o bottom temperature & 0.041 & 0.204 & $<0.01$ \\
\hline w/o depth & 0.042 & 0.205 & $<0.01$ \\
\hline w/o sediment & 0.032 & 0.179 & $<0.01$ \\
\hline Fall GMM, sample-based HSI & 0.039 & 0.199 & $<0.01$ \\
\hline w/o bottom temperature & 0.038 & 0.194 & $<0.01$ \\
\hline w/o depth & 0.043 & 0.208 & $<0.01$ \\
\hline w/o sediment & 0.030 & 0.173 & $<0.01$ \\
\hline
\end{tabular}


Figure 1. Density field plots from the delta-generalized spatio-temporal model. Red indicates areas of higher abundance, blue indicates areas of lower abundance. Pearson residual plots of the

737 density field estimates from the delta-generalized spatio-temporal model.

(a) Spring, BTS \& LLS
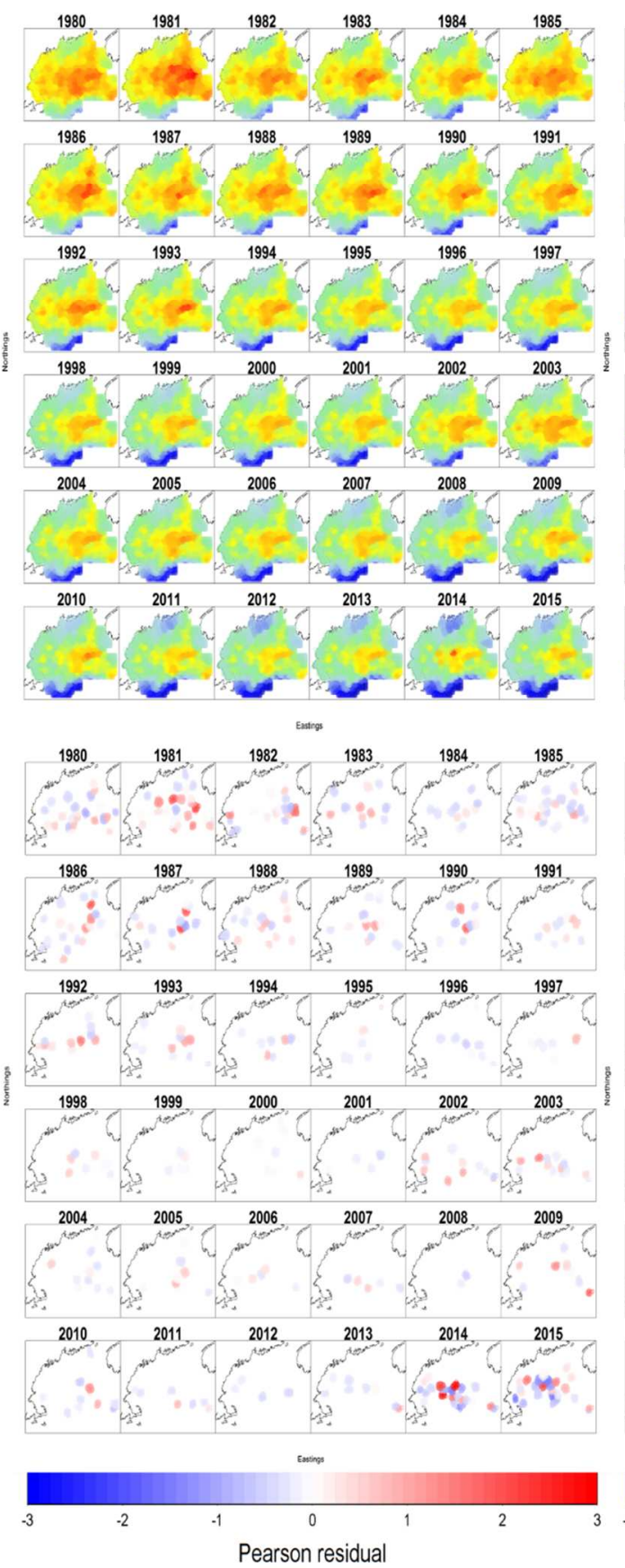

(b) Fall, BTS \& LLS
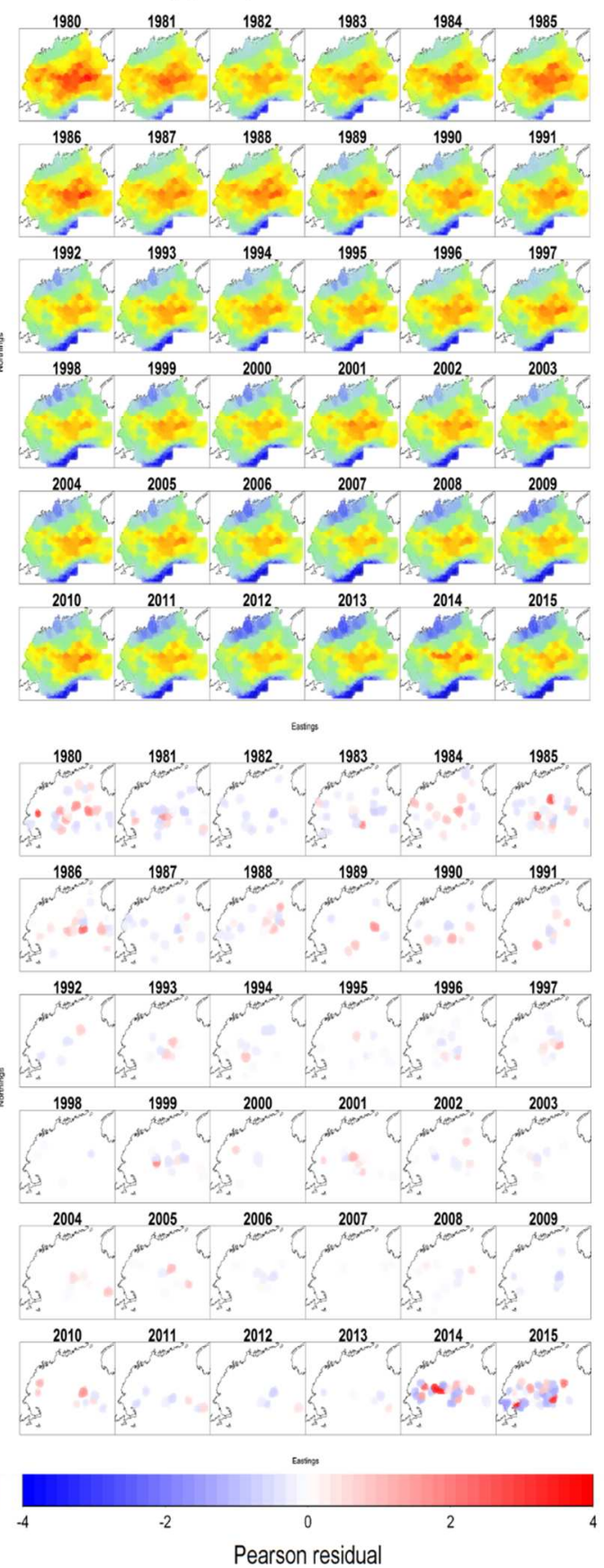
740 Figure 2. Linear regression of simulated and observed mean, seasonal bottom temperature.

741 NECOFS simulated, seasonal mean temperatures (x-axis) compared to instantaneous observed 742 bottom temperature from the NEFSC BTS (y-axis), when recorded on the survey.

Spring Bottom Temperature

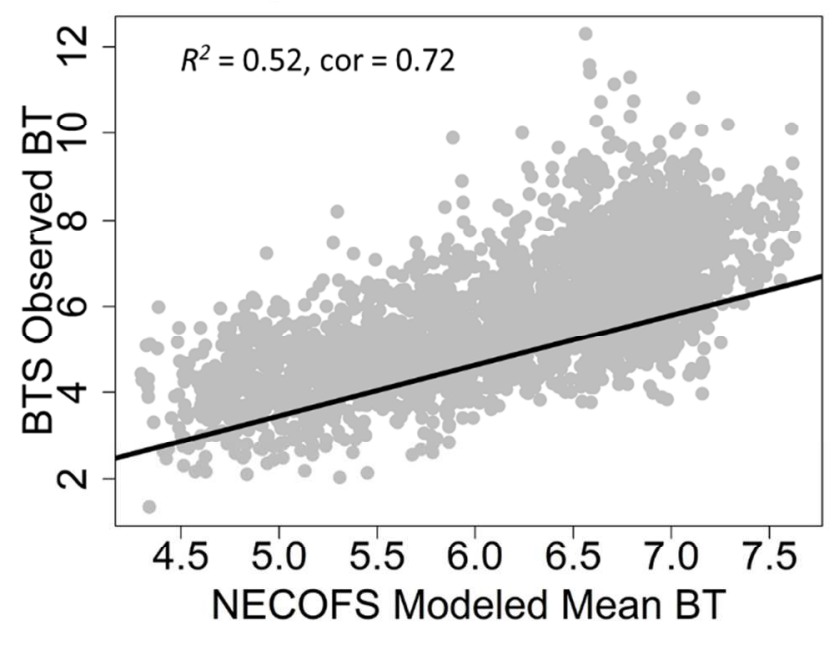

Fall Bottom Temperature

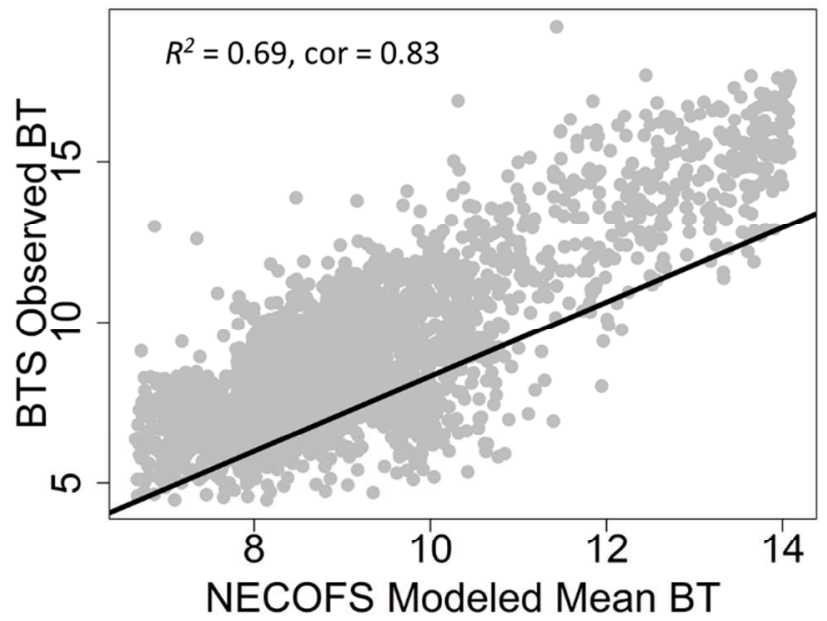


756 Figure 3. Linear regression of simulated and observed mean depths. GEBCO simulated depths

757 (x-axis) compared to mean observed bottom depths from the NEFSC BTS (y-axis) recorded on 758 the survey.

759

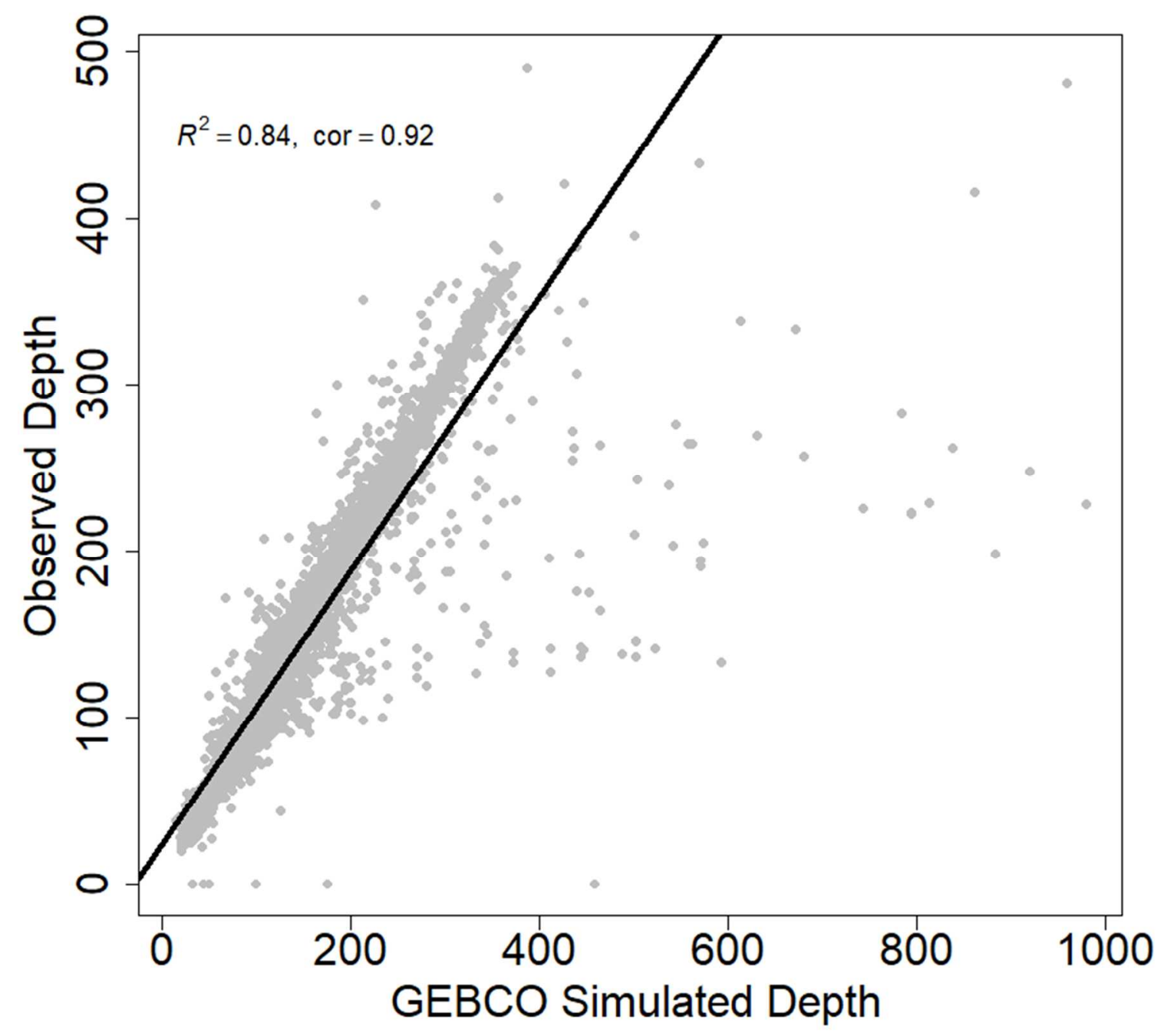


761 Figure 4. Comparing model-based and sample-based suitability index curves for cusk. For these

762

763

764

765

766

767

768

(a) Model-based HSI
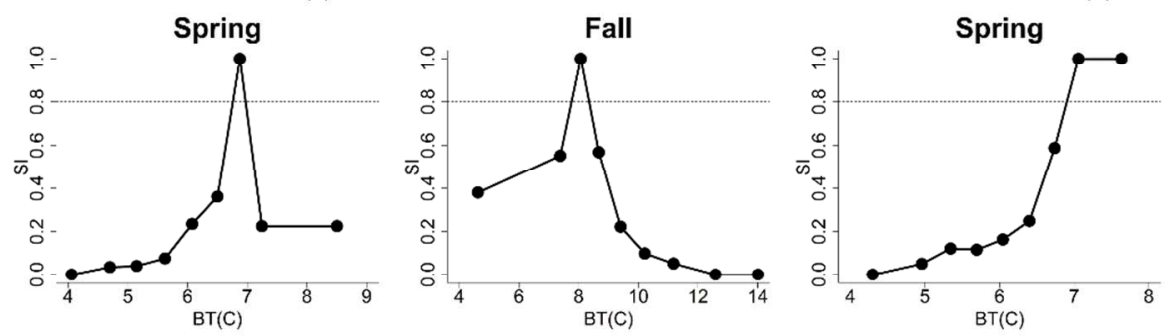

(b) Sample-based HSI

LLS; (b) sample-based abundance SIs derived from the BTS. Sediment types include: bedrock (br), gravel (gr), gravelly sediment (gr-sd), sand (sd), 33\% sand, silt, and clay (sd/st/cl), 25\% $50 \%$ sand with clay and silt (sd-cl/st), > 75\% sand with silt and clay (sd-st/clay), 50-75\% clay with silt with sand (cl-st/sd), clay (cl) (Poppe et al. 2003).
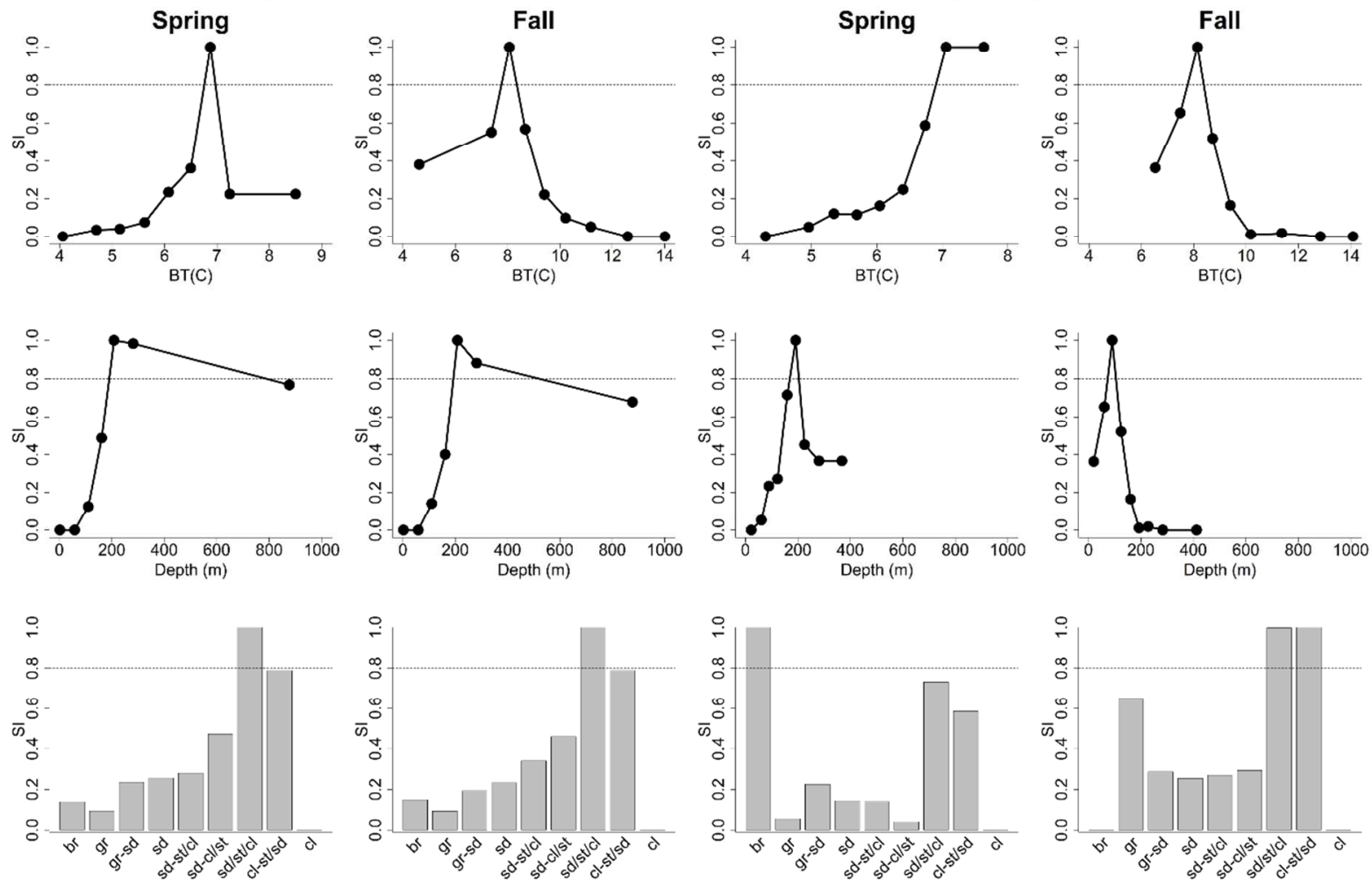
774 Figure 5. Spring and fall BTS positive catches of cusk by sediment type. Low catch rates of cusk 775 at complex sediment types is likely to lead to biased sediment suitability index (SI) estimates.

776 Sediment histograms are ordered from coarsest sediment (bedrock) to the finest sediment (clay).

777 Sediment types include: bedrock (br), gravel (gr), gravelly sediment (gr-sd), sand (sd), 33\% sand, 778 silt, and clay (sd/st/cl), 25\% - 50\% sand with clay and silt ( $\mathrm{sd}-\mathrm{cl} / \mathrm{st}),>75 \%$ sand with silt and 779 clay (sd-st/clay), 50-75\% clay with silt with sand (cl-st/sd), clay (cl) (Poppe et al. 2003).

780

781
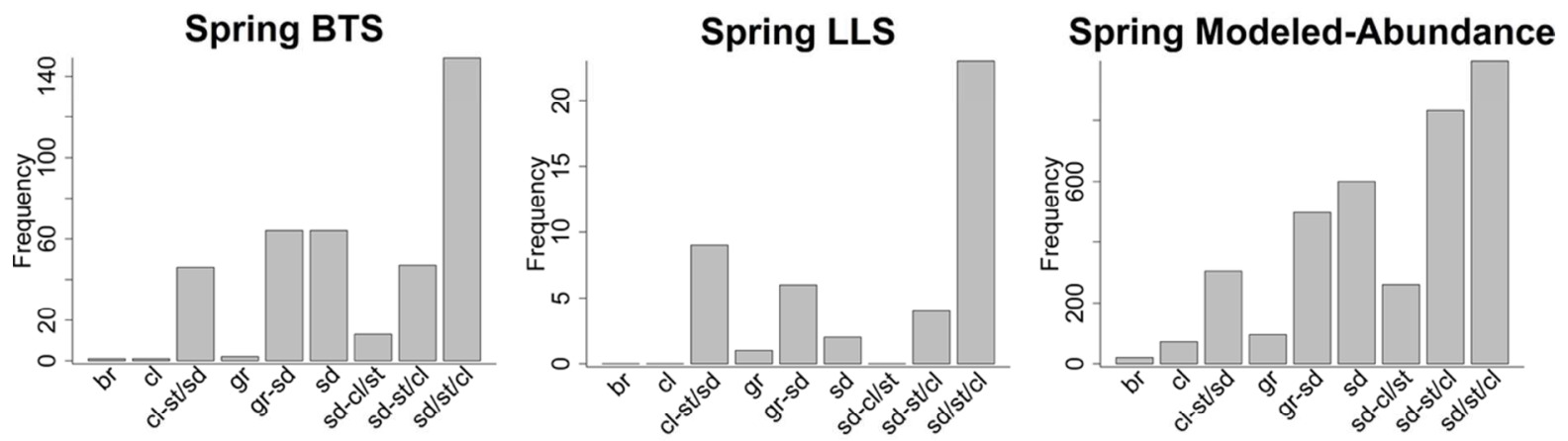

Fall BTS
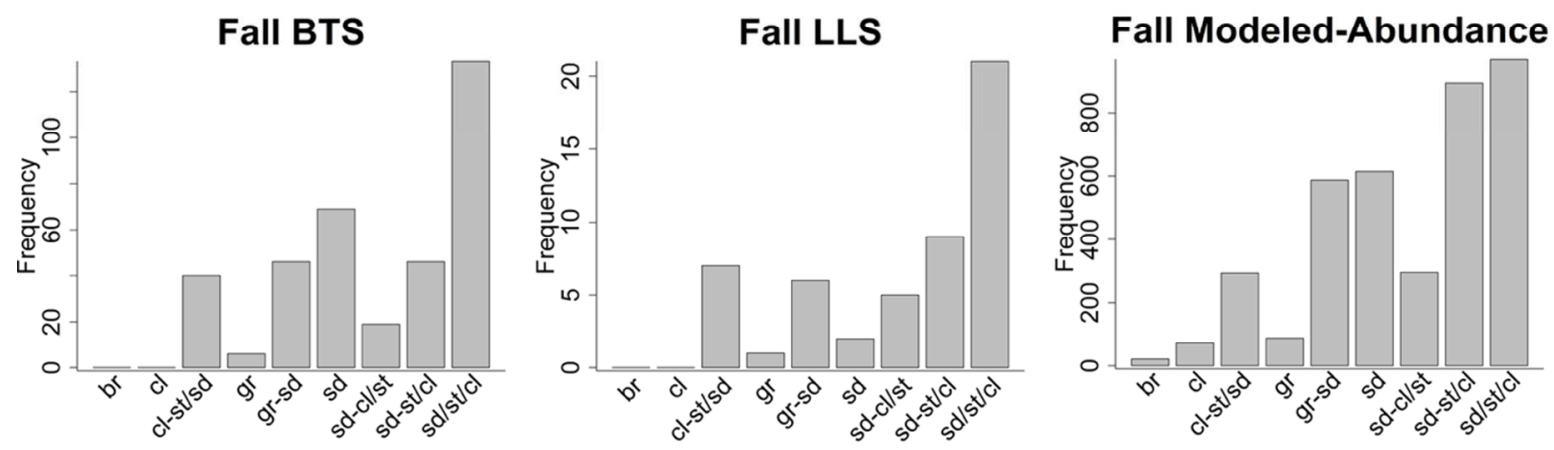

783

784

785

786

787 
788 Figure 6. Sampling frequency of depths in the NEFSC spring and fall bottom trawl surveys.
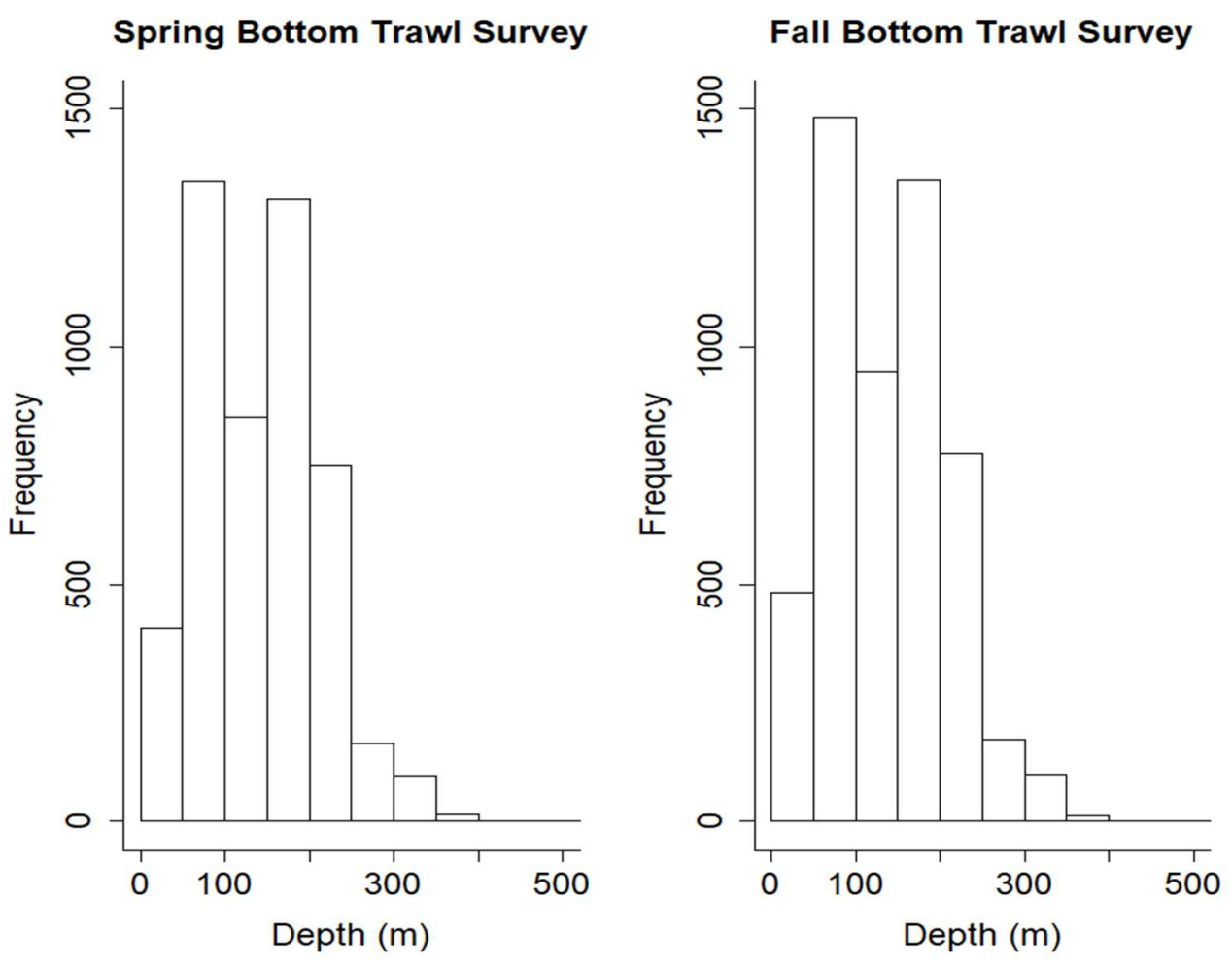
801 Figure 7. Size distribution of cusk caught in the NEFSC longline (2014 - 2015) and trawl 802 surveys (1980 - 2015), spring and fall combined.
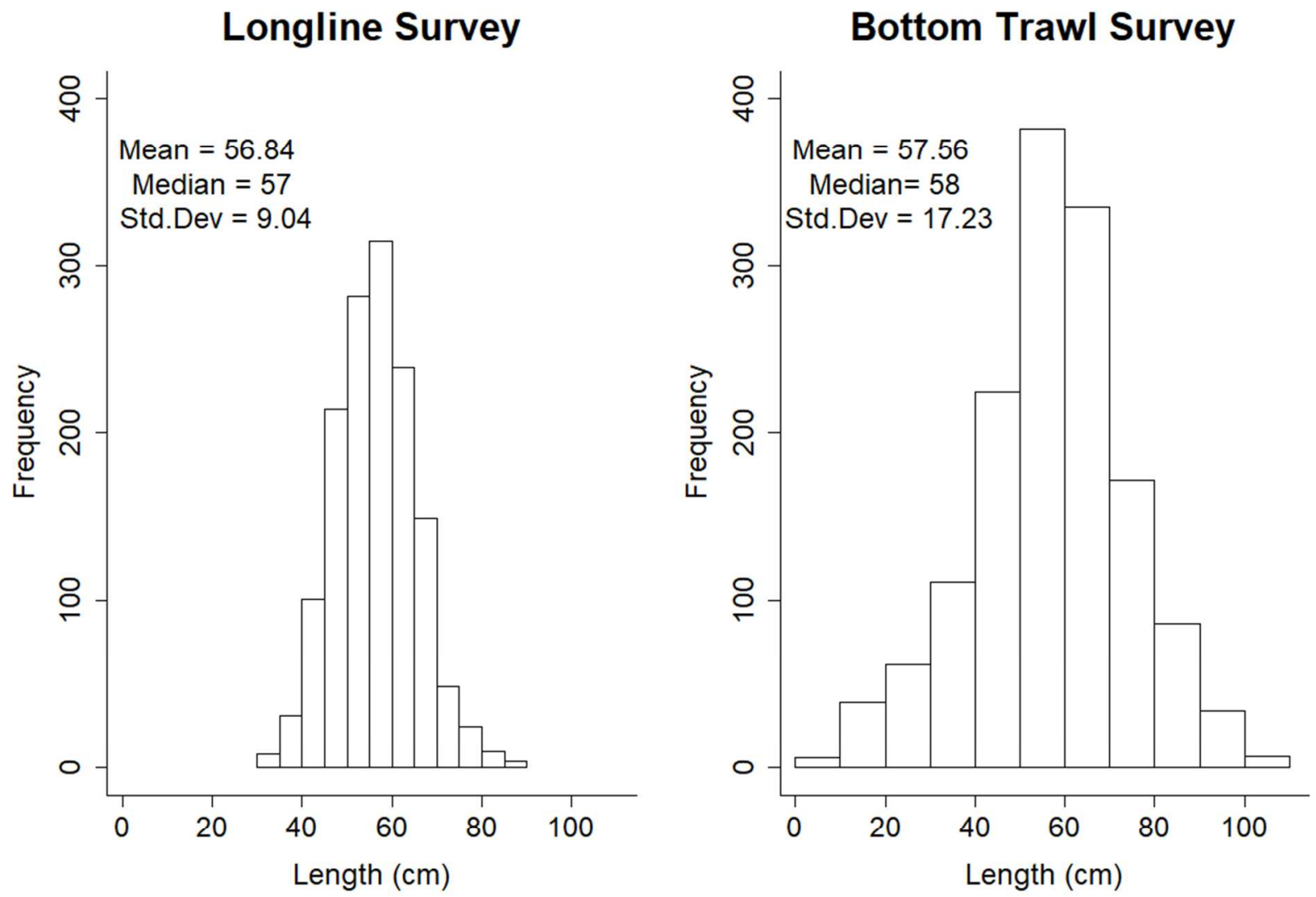

803

804

805

806

807

808

809

810

811 
812 Figure 8. Habitat Suitability Index maps for cusk. These HSI maps were derived from the model-

813 based density estimates using data from both the bottom trawl and longline surveys. High habitat

814 quality (red) is mostly offshore for both the spring and fall and lower habitat quality (dark blue)

815 is mostly inshore and around Georges Bank. Positive catch rates from the BTS (circles) and the

816 LLS (triangles) are used to validate model predictions of cusk habitat quality. The size of the

817 circle or triangle indicate catch rates, with smaller circles/triangles indicating lower catch rates

818 compared to the larger circle/triangle.
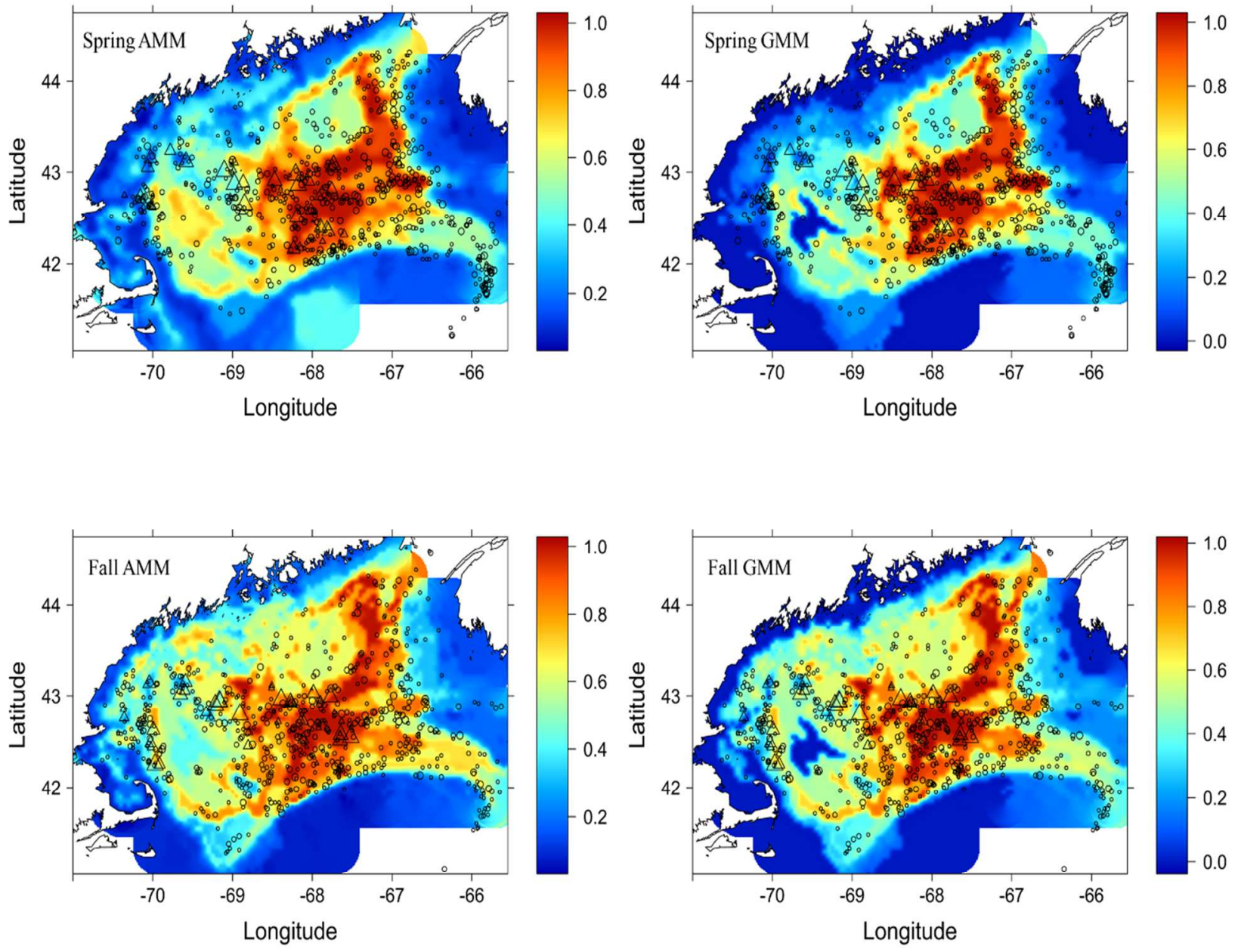


\section{Appendix I. Developing density estimates and abundance indices utilizing multiple gear}

\section{2 types}

3 This study, in part, set out to determine if data from two different gear types could be combined

4 to develop indices of abundance for data limited species using the delta-GLMM. Positive catch

5 rates in the model are a function of area swept. However, this study combined two types of

6 surveys with two different concepts of 'area swept'. The bottom trawl survey area swept is

7 considered a standardized volume that is a function of the width of the doors and trawl speed.

8 Longline surveys do not have a standardized area fished.

9

The delta-GLMM first estimates the probability of presence then estimates positive catch rates in the second stage. The first and second stage of the model is fit using the survey data. The first stage of the model is fit as a function of annual average density, catchability, and spatial and spatial temporal random effects estimated from the nearest knot. The second stage of the model is fit given all of the same parameters as a function of area swept. Catchability is then removed, and the underlying species density is predicted at each knot. Total abundance across the domain is calculated as:

$$
\hat{b}_{t}=\sum_{j=1}^{n_{j}} a_{j} \operatorname{logit}^{-1}\left(d_{T_{(i}}^{(p)}+\omega_{J_{(i)}}^{(p)}+\varepsilon_{J_{(i)}, T_{(i)}}^{(p)}\right) \exp \left(d_{T_{(i)}}^{(\lambda)}+\omega_{J_{(i)}}^{(\lambda)}+\varepsilon_{J_{(i)}, T_{(i)}}^{(\lambda)}\right)
$$

Full model details can be found in Thorson et al. (2015).

To test if catchability within the delta-GLMM was capable of accounting for differences of gear types, different estimates of area fished were evaluated for the LLS. The distance between the beginning and end of a longline set were known but the bait plume around the longline was not known. Bait plume is a function of current speed and direction, bait type, and soak duration, 
24 which sets the range over which the bait can be detected, as well as factors influencing the range 25 over which fish will respond to detected bait; the fish response factors include length of food 26 deprivation (i.e., hunger), fish size, and swimming speed (Løkkeborg et al. 1995; Zhou et al.

27 2014). These specifics are not known in fisheries surveys, and feeding response to bait plumes

28 has not been measured for cusk. Without knowing the details necessary to estimate the bait 29 plume, three values of $b$ were tested based on the estimates for moderate food deprivation in 30 Løkkeborg et al. (1995). A minimum, medium, and maximum value of $b(280 \mathrm{~m}, 560 \mathrm{~m}, 1121 \mathrm{~m}$ 31 respectively) were used to test the sensitivity of abundance estimates to longline area fished.

A total of six model-based density fields with different configurations of values for $b$ were 34 estimated for spring and fall. Each season had three models that incorporated both the BTS and LLS using the three values of $b$ (i.e., $0.28 \mathrm{~km}, 0.56 \mathrm{~km}$, and $1.12 \mathrm{~km}$ ). The resulting estimates for area fished varied by an order of magnitude (less than $0.5 \mathrm{~km}^{2}$ when $b=0.28 \mathrm{~km}$ and up to

$372.0 \mathrm{~km}^{2}$ when $\left.b=1.121 \mathrm{~km}\right)$. Three catchability parameters were estimated to account for the 2009 protocol changes in the BTS. The estimated abundance index for the three models in the spring and three in the fall were unchanged with changes in the value of $b$. AICs for all three values of $b$ were unchanged for the different model runs in both the spring (3837) and the fall 41 (3676). Four model-based abundance indices were derived using only the spring and fall bottom trawl 44 survey (BTS) then combining the BTS and the spring and fall longline survey (LLS). Two catchability coefficients were defined for before and after the 2009 protocol changes to the BTS, 46 treating the survey as two surveys within each season with no temporal overlap. Therefore, 
47 models with data from both survey programs estimated three catchability parameters and models

48 that included only the BTS estimated two. The resulting abundance estimates do not vary in

49 relative trend from the abundance estimates using the combined surveys (Figure A1). Abundance

50 was high in 1980 - 1981 with a decline to persistent low levels since 2005 (Figure A1).

51 However, there is a slight difference in trends for the two most recent years of the time series

52 (2014-2015) when the longline survey is added (Figure A1). All model-based abundance indices

53 show a decrease in cusk abundance over the time series $(1980-2015)$ for both seasons. Pearson

54 residual plots suggest there is no significant spatio-temporal pattern in the residuals (Figure A2).

55

56 When catchability was not estimated for the LLS, the annual abundance index was inconsistent

57 and highly variable during the exploratory phase of this study (results not shown). The delta-

58 GLMM could account for differences in catchability between the LLS and BTS, indicating this is

59 an effective method of incorporating multiple surveys with different gear types to estimate

60 abundance indices, even without accurate bait plume measures for a longline survey.

61

62

63

64

65

66

67

68 
70 Figure A1. Brosme brosme model-based abundance indices. Derived from the both the NEFSC

71 bottom trawl survey and longline survey combined and the NEFSC bottom trawl survey only.

72 The spring and fall model-based abundance indices accounted for spatial and spatio-temporal

73 randomness. Error bars are standard deviations from the annual mean.

74

75

76
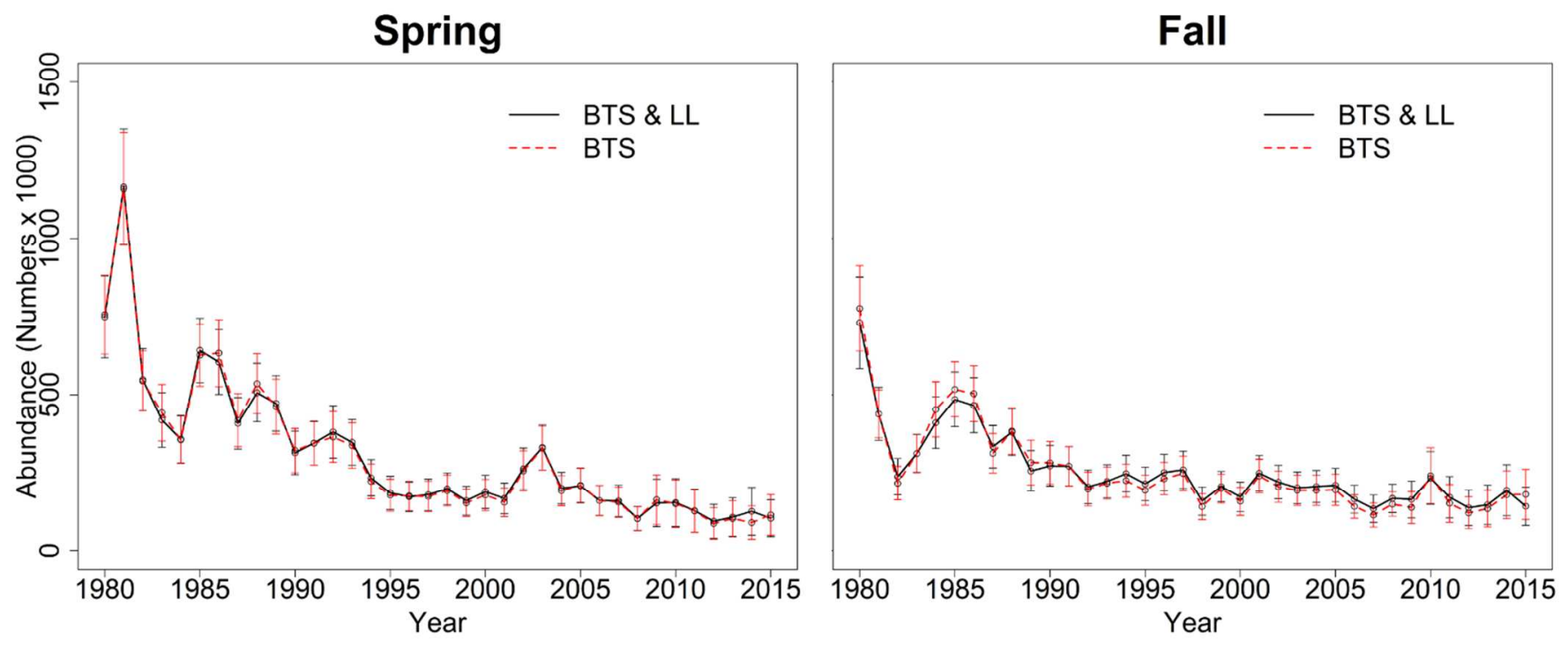

77

78

79

80

81

82

83

84

85 
87 areas of higher abundance, blue indicates areas of lower abundance. Pearson residual plots of the

(a) Spring, BTS

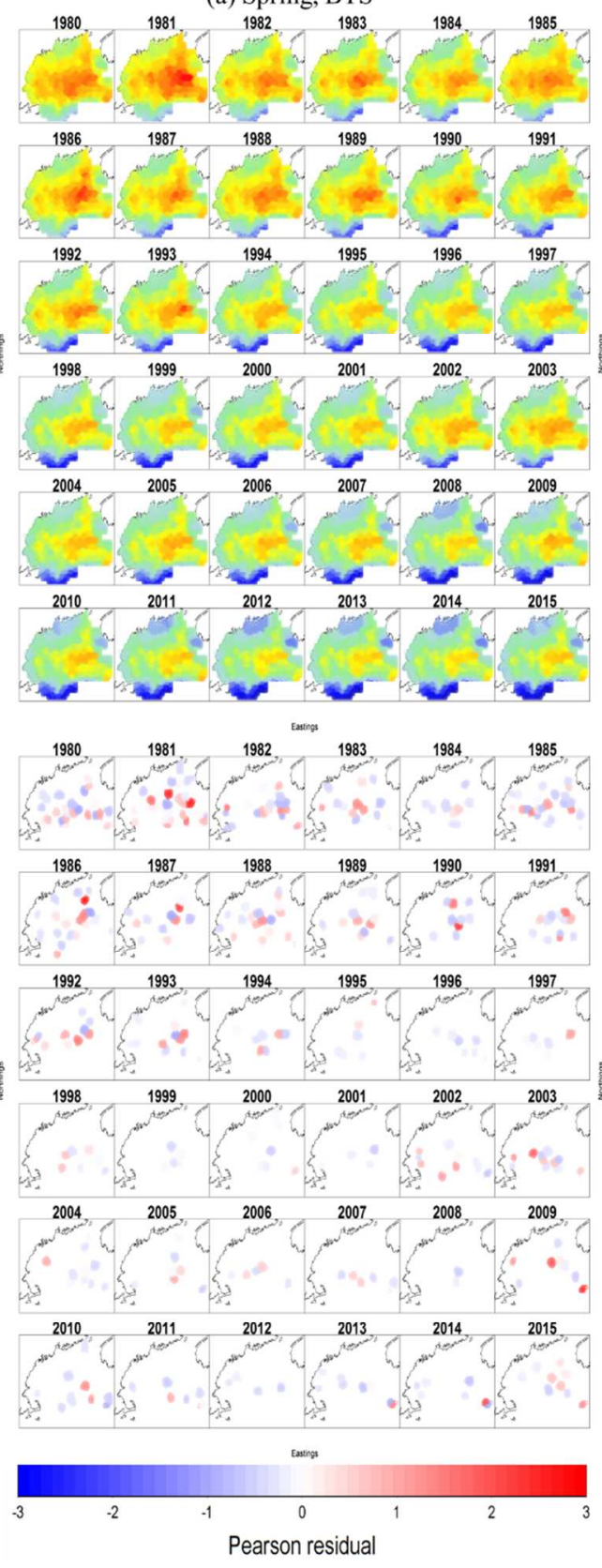

(b) Fall, BTS
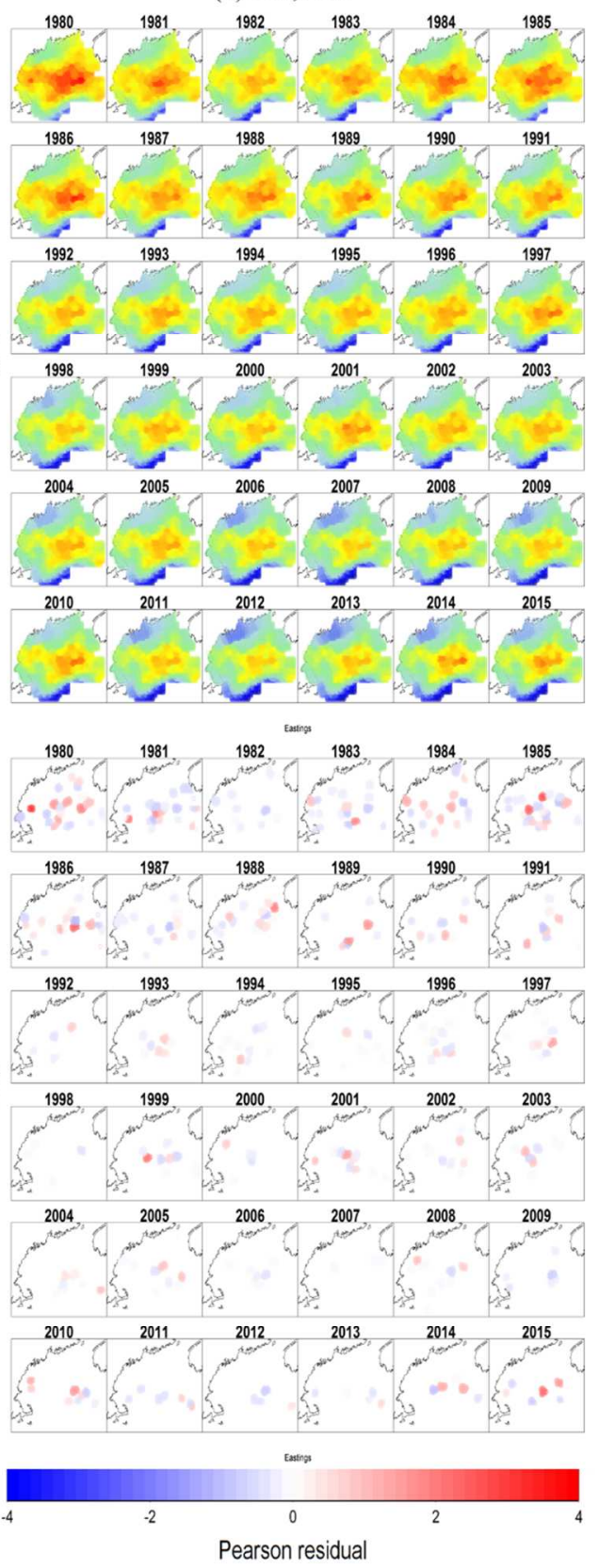
